\title{
Introduction to Random Walks on Noncommutative Spaces
}

\author{
Philippe Biane
}

Abstract We introduce several examples of random walks on noncommutative spaces and study some of their probabilistic properties. We emphasize connections between classical potential theory and group representations.

\section{Introduction}

Whereas random walks form one of the most investigated class of stochastic processes, their noncommutative analogues have been studied only recently. In these lectures I will present some results on random walks which take their values in noncommutative spaces. The notion of a noncommutative space has emerged progressively from the development of quantum physics, see e.g. [C]. The key idea is to consider not the space itself but the set of real, or complex functions on it. For a usual space, this forms an algebra, which is commutative by nature. A noncommutative space is given by a noncommutative (usually complex) algebra which is to be thought of as the algebra of complex functions on the space. We shall explain this idea in more details in section 2 , and in particular define noncommutative probability spaces. Once noncommutative spaces have been defined in this way it is easy to define random variables, and stochastic processes taking their values in these spaces. Rather than starting an abstract theory, these lectures will consist mainly in a collection of examples, which I think show that this notion is interesting and worth studying. We shall begin with the most simple stochastic process namely the Bernoulli random walk. We shall show how to quantize it in order to construct the quantum Bernoulli random walk. Simple as it is this

\footnotetext{
P. Biane

Institut d'électronique et d'informatique Gaspard-Monge, 77454 Marne-la-Vallée Cedex 2, France

e-mail: biane@univ-mlv.fr 
noncommutative stochastic process exhibits quite deep properties, related to group representation and potential theory. Actually interpreting it as a random walk with values in a noncommutative space, the dual of $S U(2)$, we will be lead to define random walks with values in duals of compact groups. The study of such random walks in the case of special unitary groups uncovers connections with potential theory, in particular with the Martin boundary. We will investigate more on these connections. From the Bernoulli random walk we can take limit objects, as in the central limit theorem. One of these objects is a noncommutative Brownian motion which we construct as a family of operators on a Fock space and interpret then as a continuous time stochastic process with independent increments, with values in the dual of the Heisenberg group. We then extend this construction to more general noncompact locally compact groups. Finally we will also start to consider quantum groups in the last section.

The next section consists in preliminaries about $C^{*}$ and von Neumann algebras and noncommutative spaces.

\section{Noncommutative Spaces and Random Variables}

\subsection{What are Noncommutative Spaces?}

The random walks that we are going to study take their values in noncommutative spaces, so we should start by making this notion more precise. In many parts of mathematics, one studies spaces through the set of functions defined on them. There can be many kind of functions, e.g. measurable, integrable, continuous, bounded, differentiable, and so on. Each property of the functions reflects a property of the space on which they are defined. Sometimes, in probability theory for example, one is even not interested at all in the space, but only in the functions themselves, the random variables. Also very often the set of complex functions considered determines completely the underlying space. This is the case for example for compact topological spaces, determined by their algebra of continuous functions, or differentiable manifolds which are determined by their smooth functions. The common feature shared by these situations is that all these spaces of complex valued functions are commutative algebras. It has been realized, since the beginning of quantum mechanics that one can obtain a better description of nature by relaxing this commutativity hypothesis. Henceforth we shall consider a non commutative space as given by a complex algebra, which plays the role of space of functions on the space. We will see many examples throughout these lectures. Some algebras may come equipped with a supplementary structure, for example an antilinear involution, a norm, a preferred linear form, a topology, etc... Actually most of the times these algebras will be algebras 
of operators on some complex Hilbert space $H$, and the involution will be given by the adjoint operation. We shall decribe the kind of algebras we will consider, mainly $C^{*}$ and von Neumann algebras. We will use the language and some basic results in the theory of these objects, but we will need no deep knowledge of them. We will only assume that the reader is familiar with the spectral theorem for selfadjoint operators on a Hilbert space. We refer for example to the treatises [D1], [D2] or [T] for more details.

\section{2 $C^{*}$ Algebras}

A $C^{*}$ algebra is a normed $*$-algebra which is isometric with a subalgebra of the algebra $B(H)$ of all bounded operators on some complex Hilbert space $H$, stable under taking the adjoint, and closed for the operator norm topology. Elements in a $C^{*}$-algebra of the form $a a^{*}$ for some $a \in A$ are called positive. Positive elements are exactly the selfadjoint positive operators which belong to the algebra.

Let $X$ be a locally compact topological space, then the algebra of complex continuous functions on $X$, vanishing at infinity, is a $C^{*}$-algebra, and the famous Gelfand-Naimark theorem states that any commutative $C^{*}$-algebra is isomorphic to such an algebra. The topological space is compact if and only if the algebra has a unit, and there is a one to one correspondence between the points of the space and the characters of the algebra, that is, the continuous algebra homomorphisms with values in the complex numbers, or equivalently with the maximal closed ideals, therefore the space is unique up to homeomorphism and can be recovered from the algebra. It is usually denoted by $\operatorname{spec}(A)$ if $A$ is the $C^{*}$-algebra. Thus we should think of a $C^{*}$-algebra as providing the algebra of continuous functions on some noncommutative space. Note that $C^{*}$ algebras are closed under continuous functional calculus, namely if $a$ is a self-adjoint element in a $C^{*}$ algebra, and $f$ a continuous functions on its spectrum, then the operator $f(a)$ also belongs to the algebra $C$. This can be easily seen by approximating uniformly $f$ by polynomials on the spectrum of $a$.

If $A \subset B(H)$ is a $C^{*}$-algebra, then the multiplier algebra $M(A)$ of $A$ is the set of all operators $x$ such that $x A \subset A$ and $A x \subset A$. It is a $C^{*}$ algebra with a unit, containing $A$. It coincides with $A$ if and only if $A$ has a unit. If $A$ is abelian, then $M(A)$ is just the algebra of all bounded continuous functions on $\operatorname{spec}(A)$. In the noncommutative situation, it corresponds to the Stone-Cech compactification of the topological space underlying the algebra.

Continuous positive linear functionals on a $C^{*}$ algebra play the role of positive bounded measures. Here positivity for a functional means that it is positive on positive elements. Again in the commutative case, by Riesz' theorem, such linear functionals correspond to finite positive Borel measures on the underlying topological space. Positive linear functionals of norm one 
are called states, and correspond to probability measures in the commutative case. A large supply of states is given by unit vectors in the Hilbert space on which the $C^{*}$ algebra acts. Indeed any such vector $\psi$ defines a state by the formula

$$
\omega_{\psi}(a)=\langle a \psi, \psi\rangle \quad a \in A
$$

Given a self adjoint element $a$ in a $C^{*}$ algebra $A$, and a state $\sigma$ on $A$, there exists a unique measure on $\mathbb{R}$, with compact support, such that

$$
\sigma(f(a))=\int f(x) d \mu(x) \quad \text { for all continuous } f \text { on } \mathbb{R} \text {. }
$$

The support of this measure is included in the spectrum of $a$. The GNS construction assigns to every $C^{*}$ algebra, with a continuous positive linear functional $\sigma$, a representation of the algebra on a Hilbert space. A linear functional is called tracial if for any $a, b \in A$ one has $\tau(a b)=\tau(b a)$.

Each continuous map between topological spaces $f: X \rightarrow Y$ gives rise to a continuous algebra morphism $\Phi_{f}: C_{0}(Y) \rightarrow C_{0}(X) ; h \mapsto h \circ f$, and conversely any such algebra morphism comes from a continuous map, therefore one can think of a homomorphism between $C^{*}$ algebras as a continuous map between the underlying noncommutative spaces (with the direction of the arrows reversed). One must note however that there may exist very few morphisms between two $C^{*}$ algebras. For example there does not exist any nonzero homomorphism from the finite dimensional $C^{*}$ algebra $M_{n}(\mathbb{C})$ to $M_{m}(\mathbb{C})$ if $n>m$. Indeed this is a purely algebraic fact, since $M_{n}(\mathbb{C})$ is a simple algebra, if a homomorphism from $M_{n}(\mathbb{C})$ is not injective, then it must be 0 .

\section{3 von Neumann Algebras}

Let $S$ be a subset of $B(H)$, then its commutant $S^{\prime}$ is the set of bounded operators which commute with every element of $S$. A von Neumann algebra is a subalgebra of $B(H)$ which is closed under under taking the adjoint, and is equal to its bicommutant, i.e. the commutant of its commutant. By the von Neumann bicommutant theorem the von Neumann algebras are the *-subalgebra of $B(H)$, containing the identity operator, and closed for the strong topology. Since the strong topology is weaker than the operator norm topology any von Neumann algebra is also a unital $C^{*}$ algebra, although generally too large to be interesting as such.

A von Neumann algebra is closed under Borel functional calculus, namely if $a \in M$ is self-adjoint and $f$ is a bounded Borel function on the spectrum of $a$, then $f(a)$ belongs to $M$, and again, the same is true for $f\left(a_{1}, \ldots, a_{n}\right)$ where $a_{1}, \ldots, a_{n}$ are commuting selfadjoint operators in the von Neumann algebra, and $f$ is a bounded Borel function defined on the product of their spectra. 
The nuance between $C^{*}$ algebras and von Neumann algebras can be grasped by looking at the commutative case. Indeed commutative von Neumann algebras correspond to measure spaces, more precisely any commutative von Neumann algebra is isomorphic to the algebra $L^{\infty}(X, m)$ where $X$ is a measure space and $m$ a positive measure (the algebra actually depends only on the class of the measure). This statement can be seen as a reformulation of the spectral theorem for commuting self-adjoint operators on a Hilbert space. Therefore it is natural to think of von Neumann algebras as "algebras of noncommutative random variables". A normal state on a von Neumann algebra is a positive linear form which is continuous for the $\sigma$-weak topology and takes the value 1 on the unit. It corresponds, in the commutative case, to a probability measure, which is absolutely continuous with respect to the measure $m$. We shall sometimes call a von Neumann algebra, with a normal state, a "non commutative probability space".

A weight on a von Neumann algebra is a map $\varphi$ from the cone of positive elements of the von Neumann algebra to $[0,+\infty]$, which is additive, and homogeneous, i.e. $\varphi(\lambda x)=\lambda \varphi(x)$ for $x$ positive and real $\lambda>0$. A weight is called normal if $\sup _{i \in I} \varphi\left(x_{i}\right)=\varphi\left(\sup \left(x_{i}\right)_{i \in I}\right)$ for every bounded increasing net $\left(x_{i}\right)_{i \in I}$. Coming back to the commutative case, weights are positive, possibly unbounded measures, in the measure class of $m$. A weight $\mu$ is called finite if $\mu(1)<\infty$, in this case $\mu$ is a multiple of a state.

Given a selfadjoint element, $a \in M$ and a normal state $\sigma$ on $M$, we denote by $\mu_{a}$ the distribution of $a$, namely the measure such that

$$
\sigma(f(a))=\int f(x) d \mu_{a}(x)
$$

for all bounded Borel functions on $\operatorname{spec}(a)$. More generally if $a_{1}, \ldots, a_{n}$ is a family of commuting self-adjoint operators in $M$, their joint distribution is the unique probability measure $\mu_{a_{1}, \ldots, a_{n}}$ on $\mathbb{R}^{n}$ such that

$$
\sigma\left(f\left(a_{1}, \ldots, a_{n}\right)\right)=\int f(x) d \mu_{a_{1}, \ldots, a_{n}}(x)
$$

for all bounded Borel function $f$ on $\mathbb{R}^{n}$.

Let $N \subset M$ be a von Neumann subalgebra, and $\sigma$ a state on $M$, then a conditional expectation of $M$ onto $N$ is a norm one projection $\sigma(. \mid N)$ such that $\sigma(a \mid N)=a$ for all $a \in N, \sigma(a b c \mid N)=a \sigma(b \mid N) c$ for all $a, c \in N, b \in M$, and $\sigma(\sigma(b \mid N))=\sigma(b)$ for all $b \in M$. Given $M, N$ and $\sigma$, such a map need not exist, but it always exists, and is unique, if $\sigma$ is tracial.

We will consider spatial tensor products of von Neumann algebras. If $A \subset B(H)$ and $B \subset B(K)$ are two von Neumann algebras, their algebraic tensor product acts on the Hilbert space $H \otimes K$, and the spatial tensor product of $A$ and $B$ is defined as the von Neumann algebra generated by this tensor product. Given an infinite family of von Neumann algebras $\left(A_{i} ; i \in I\right)$ equipped with normal states $\omega_{i}$ it is possible to construct an infinite tensor 
product $\otimes_{i}\left(A_{i}, \omega_{i}\right)$ which is a von Neumann algebra with a state $\otimes_{i} \omega_{i}$. One considers operators of the form $\otimes_{i \in I} a_{i}$ where $a_{i} \in A_{i}$ and $a_{i} \neq I d$ only for a finite number of $i \in I$. These generate an algebra, which is the algebraic tensor product of the $A_{i}$. One can define a positive linear functional on this algebraic tensor product by $\omega\left(\otimes_{i \in I} a_{i}\right)=\prod \omega_{i}\left(a_{i}\right)$. The GNS construction then yields a Hilbert space $H$, with a pure state on $B(H)$, and the von Neumann algebra tensor product is the von Neumann algebra in $B(H)$ generated by this algebraic tensor product.

\subsection{Random Variables, Stochastic Processes with Values in some Noncommutative Space}

Given a von Neumann algebra $M$ equipped with a normal state $\sigma$, and a $C^{*}$ algebra $C$, a random variable with values in $C$ (or, more appropriately, in the noncommutative space underlying $C$ ), is a norm continuous morphism from $C$ to $M$. The distribution of the random variable $\varphi: C \rightarrow M$ is the state on $C$ given by $\sigma \circ \varphi$. If the algebra $C$ is commutative, then it corresponds to some topological space, and the state $\sigma \circ \varphi$ to a probability measure on this space. When $C=C_{0}(\mathbb{R})$ there exists a self-adjoint element $a \in M$ such that $\varphi(f)=f(a)$ for all $f \in C$, and we are back to the situation in the preceding section, where the distribution of $a$ was defined. We will call the state $\sigma \circ \varphi$ the distribution of the random variable $\varphi$. More generally a family of random variables with values in some noncommutative space, indexed by some set, is a stochastic process.

If $A$ and $B$ are two $C^{*}$-algebras, a positive map $\Phi: A \rightarrow B$ is a linear map such that $\Phi(a)$ is positive for each positive $a \in A$. When $A$ and $B$ are commutative, thus $A=C_{0}(X)$ and $B=C_{0}(Y)$, such a linear map can be realized as a measure kernel $k(y, d x)$ where for each $y \in Y$ one has a finite positive measure $k(y, d x)$ on $X$. If $A$ and $B$ are unital, and $\Phi(I)=I$ then this kernel is a Markov kernel, i.e. all measures are probability measures. Thus we see that the generalization of a Markov kernel to the non-commutative context can be given by the notion of positive maps. It turns out however that this notion is slightly too general to be useful and it is necessary to restrict oneself to a particular class called completely positive maps.

Definition 2.1. linear map between two $C^{*}$ algebras $A$ and $B$ is called completely positive if, for all $n \geq 0$, the map $\Phi \otimes I d: A \otimes M_{n}(\mathbb{C}) \rightarrow B \otimes M_{n}(\mathbb{C})$ is positive. It is called unit preserving if furthermore $\Phi(I d)=I d$.

We shall consider semigroups of unit preserving, completely positive maps on a $C^{*}$ algebra $C$. These will be indexed by a set of times which will be either the nonnegative integers (discrete times) or the positive real line (continuous time). Thus a discrete time semigroup of unit preserving, completely positive maps on a $C^{*}$ algebra $C$ will be a family $\left(\Phi_{n}: C \rightarrow C\right)_{n \geq 0}$ of completely 
positive maps, such that $\Phi_{n} \circ \Phi_{m}=\Phi_{n+m}$. In continuous time we will have a family $\left(\Phi_{t}\right)_{t \in \mathbb{R}_{+}}$which satisfies $\Phi_{t} \circ \Phi_{s}=\Phi_{t+s}$. In the discrete time setting one has $\Phi_{n}=\left(\Phi_{1}\right)^{n}$ and the semigroup is deduced from the value at time 1 . We shall denote generally the time set by $T$ when we do not specify whether we are in discrete or continuous time.

Definition 2.2. Let $C$ be a $C^{*}$ algebra, then a dilation of a semigroup $\left(\Phi_{t}\right)_{t \in T}$ of completely positive maps on $C$ is given by a von Neumann algebra $M$, with a normal state $\omega$, an increasing family of von Neumann subalgebras $M_{t} ; t \in T$, with conditional expectations $\omega\left(. \mid M_{t}\right)$, and a family of morphisms $j_{t}: C \rightarrow(M, \omega)$ such that for any $t \in T$ and $a \in C$, one has $j_{t}(a) \in M_{t}$ and for all $s<t$

$$
\omega\left(j_{t}(a) \mid M_{s}\right)=j_{s}\left(\Phi_{t-s}(a)\right)
$$

A dilation of a completely positive semigroup is the analogue in noncommutative probability of a Markov process, and the equation (2.1) expresses the Markov property of the process: the conditional expectation of the future on the past is a function of the present.

Given a completely positive semigroup and an initial state, a dilation always exists $[\mathrm{S}]$.

Consider a completely positive semigroup on a $C^{*}$ algebra $C$, and let $B \subset C$ be a commutative $C^{*}$ subalgebra, thus isomorphic to $C_{0}(X)$ for some locally compact topological space $X$. If the image algebras $j_{t}(B) ; t \in T$ generate a commutative von Neumann algebra $\mathcal{N} \subset M$, then there exists a probability space $(\Omega, \mathcal{F}, P)$ such that $(\mathcal{N}, \omega) \sim L^{\infty}(\Omega, \mathcal{F}, P)$, and random variables $X_{t}: \Omega \rightarrow X$, corresponding to the morphisms $j_{t}$ restricted to $B$, which form a classical stochastic process. If furthermore the $C^{*}$ algebra $B$ is invariant by the completely positive semigroup, then this semigroup defines a Markov semigroup of transition probabilities on the space $X$, and the stochastic process $\left(X_{t}\right)_{t \in T}$ is a Markov process with these probability transitions. This remark will be at the basis of many constructions of classical stochastic processes starting from quantum ones.

Once a dilation of a completely positive semigroup is given, one can compute, for times $t_{1}<\cdots<t_{n}$, and $a_{1}, \ldots, a_{n} \in C$,

$$
\omega\left(j_{t_{1}}\left(a_{1}\right) \ldots j_{t_{n}}\left(a_{n}\right)\right)=\sigma\left(\Phi _ { t _ { 1 } } \left(a _ { 1 } \left(\Phi_{t_{2}-t_{1}}\left(a_{2}\left(\ldots \Phi_{t_{n}-t_{n-1}}\left(a_{n}\right)\right) \ldots\right)\right.\right.\right.
$$

where $\sigma=\omega \circ j_{0}$ is the initial state on $C$ (the distribution of the process at time 0 ). Observe however that when the algebras $j_{t}(C) ; t \in T$ do not commute, this condition does not specifiy the values of

$$
\omega\left(j_{t_{1}}\left(a_{1}\right) \ldots j_{t_{n}}\left(a_{n}\right)\right)
$$

when the times $t_{1}, \ldots, t_{n}$ are not ordered. We will say that two dilations $j^{(1)}, \omega_{1}$ and $j^{(2)}, \omega_{2}$, are equivalent if one has

$$
\omega_{1}\left(j_{t_{1}}^{(1)}\left(a_{1}\right) \ldots j_{t_{n}}^{(1)}\left(a_{n}\right)\right)=\omega_{2}\left(j_{t_{1}}^{(2)}\left(a_{1}\right) \ldots j_{t_{n}}^{(2)}\left(a_{n}\right)\right)
$$


where $t_{1}, \ldots, t_{n}$ is an arbitrary sequence of times (i.e. not necessarily increasing) and $a_{1}, \ldots, a_{n} \in C$. Thus if $C$ is a commutative algebra, then all commutative dilations with the same initial distribution are equivalent, but for a given semigroup of completely positive maps there may exist a lot of non equivalent dilations. Actually we shall encounter in these lectures some natural noncommutative dilations of Markov semigroups on classical spaces! An important source of such dilations comes from restrictions: if $B \subset C$ is a subalgebra and the completely positive semigroup leaves $B$ invariant, then the restriction of $\left(j_{t}\right)_{t \in T}$ to the subalgebra $B$ is a dilation of the restriction of the completely positive semigroup.

\section{Quantum Bernoulli Random Walks}

\subsection{Quantization of the Bernoulli Random Walk}

Our first example of a quantum random walk will be the quantization of the simple (or Bernoulli) random walk. This is just the random walk whose independent increments have values \pm 1 . In order to quantize it we will replace the set of increments $\{ \pm 1\}$ by its quantum analogue, namely the space of two by two complex matrices, with its structure of $C^{*}$-algebra. The subset of hermitian operators is a four dimensional real subspace, generated by the identity matrix $I$ as well as the three matrices

$$
\sigma_{x}=\left(\begin{array}{ll}
0 & 1 \\
1 & 0
\end{array}\right) \quad \sigma_{y}=\left(\begin{array}{cc}
0 & -i \\
i & 0
\end{array}\right) \quad \sigma_{z}=\left(\begin{array}{cc}
1 & 0 \\
0 & -1
\end{array}\right)
$$

The matrices $\sigma_{x}, \sigma_{y}, \sigma_{z}$ are the Pauli matrices. They satisfy the commutation relations

$$
\left[\sigma_{x}, \sigma_{y}\right]=2 i \sigma_{z} ; \quad\left[\sigma_{y}, \sigma_{z}\right]=2 i \sigma_{x} ; \quad\left[\sigma_{z}, \sigma_{x}\right]=2 i \sigma_{y} .
$$

The group $S U(2)$ acts by the automorphisms $A \rightarrow U A U^{*}$ on this $C^{*}$-algebra. We observe that this group is much larger than the group of symmetries of the two points space (which consists just of a two elements group). This action leaves the space generated by $I$ invariant, and acts by rotations on the real three dimensional space generated by the Pauli matrices. Indeed the inner product on the space of hermitian matrices $\langle A, B\rangle=\operatorname{Tr}(A B)$ is invariant by unitary conjugation.

A state $\omega$ on $M_{2}(\mathbb{C})$ is given by a positive hermitian matrix $S$ with trace 1 , by the formula

$$
\omega(A)=\operatorname{Tr}(A S) .
$$

The most general such matrix can be written as

$$
S=\frac{1}{2}\left(\begin{array}{cc}
1+u & v+i w \\
v-i w & 1-u
\end{array}\right)
$$


where $(u, v, w) \in \mathbb{R}^{3}$ satisfies $u^{2}+v^{2}+w^{2} \leq 1$. The extreme points on the unit sphere (sometimes called the "Bloch sphere" in the physics litterature), correspond to pure states, when $S$ is a projection on a one dimensional subspace. Any hermitian operator has a two-point spectrum, hence in a state $\omega$ its distribution is a probability measure on $\mathbb{R}$ supported by at most two points. In particular, for each of the Pauli matrices, its distribution in the state $\omega$ is a probability measure on $\{ \pm 1\}$, given by

$$
P\left(\sigma_{x}=1\right)=\frac{1+v}{2} \quad P\left(\sigma_{y}=1\right)=\frac{1+w}{2} \quad P\left(\sigma_{z}=1\right)=\frac{1+u}{2}
$$

Mimicking the construction of a random walk, we use the infinite product algebra $\left(M_{2}(\mathbb{C}), \omega\right)^{\otimes \mathbb{N}}$ (recall the construction of section 2.3). For each Pauli matrix we build the matrices

$x_{n}=I^{\otimes(n-1)} \otimes \sigma_{x} \otimes I^{\otimes \infty} \quad y_{n}=I^{\otimes(n-1)} \otimes \sigma_{y} \otimes I^{\otimes \infty}, \quad z_{n}=I^{\otimes(n-1)} \otimes \sigma_{z} \otimes I^{\otimes \infty}$

which represent the increments of the process. It is easy to see that, for example, the operators $x_{n}$, for $n \geq 1$, form a commuting family of operators, which is distributed, in the state $\omega^{\infty}$, as a sequence of independent Bernoulli random variables.

Then we put

$$
X_{n}=\sum_{i=1}^{n} x_{i} ; \quad Y_{n}=\sum_{i=1}^{n} y_{i} \quad Z_{n}=\sum_{i=1}^{n} z_{i} .
$$

This gives us three families of operators $\left(X_{n}\right)_{n \geq 1} ;\left(Y_{n}\right)_{n \geq 1}$ and $\left(Z_{n}\right)_{n \geq 1}$ on this space.

We observe that each of these three families consists in commuting operators, hence has a joint distribution. It is not difficult to check that this distribution is that of a Bernoulli random walk, whose increments have distribution given by the probability distributions on $\{ \pm 1\}$ of formula (3.2). The three families of operators, however do not commute. In fact using the commutation relations (3.1) one sees that for $n, m$ positive integers,

$$
\left[x_{n}, y_{m}\right]=2 i z_{m} \delta_{n m}
$$

and

$$
\left[X_{n}, Y_{m}\right]=2 i Z_{n \wedge m}
$$

as well as the similar relations obtained by cyclic permutation of $X, Y, Z$. We will call the family of triples of operators $\left(X_{n}, Y_{n}, Z_{n}\right) ; n \geq 1$ a quantum Bernoulli random walk. We shall later interpret this noncommutative process as a random walk with values in some noncommutative space, however for the moment we will study some of its properties related to the automorphisms of the algebra $M_{2}(\mathbb{C})$. 


\subsection{The Spin Process}

Because of the rotation invariance of the commutation relations (3.1), we see that for any unitary matrix $U$ we can define conjugated variables

$$
\begin{aligned}
& x_{n}^{U}=I^{\otimes(n-1)} \otimes U \sigma_{x} U^{*} \otimes I^{\otimes \infty} \\
& y_{n}^{U}=I^{\otimes(n-1)} \otimes U \sigma_{y} U^{*} \otimes I^{\otimes \infty} \\
& z_{n}^{U}=I^{\otimes(n-1)} \otimes U \sigma_{z} U^{*} \otimes I^{\otimes \infty}
\end{aligned}
$$

and

$$
X_{n}^{U}=\sum_{i=1}^{n} x_{i}^{U} ; \quad Y_{n}^{U}=\sum_{i=1}^{n} y_{i}^{U} \quad Z_{n}^{U}=\sum_{i=1}^{n} z_{i}^{U}
$$

then this new stochastic process is obtained from the original quantum Bernoulli random walk by a rotation matrix. It follows by a simple computation, using the commutation relations, that $X_{n}^{2}+Y_{n}^{2}+Z_{n}^{2}$ is invariant under conjugation, namely one has

$$
X_{n}^{2}+Y_{n}^{2}+Z_{n}^{2}=\left(X_{n}^{U}\right)^{2}+\left(Y_{n}^{U}\right)^{2}+\left(Z_{n}^{U}\right)^{2}
$$

for any unitary matrix $U$.

Lemma 3.1. For all $m, n \geq 1$ one has

$$
\left[X_{n}^{2}+Y_{n}^{2}+Z_{n}^{2}, X_{m}^{2}+Y_{m}^{2}+Z_{m}^{2}\right]=0
$$

Actually we shall prove that $\left[X_{n}, X_{m}^{2}+Y_{m}^{2}+Z_{m}^{2}\right]=0$ if $m \leq n$. This follows from the computation

$$
\begin{gathered}
{\left[X_{n}, Y_{m}^{2}\right]=\left[X_{n}, Y_{m}\right] Y_{m}+Y_{m}\left[X_{n}, Y_{m}\right]=2 i\left(Z_{m} Y_{m}+Y_{m} Z_{m}\right)} \\
{\left[X_{n}, Z_{m}^{2}\right]=\left[X_{n}, Z_{m}\right] Z_{m}+Z_{m}\left[X_{n}, Y_{m}\right]=-2 i\left(Z_{m} Y_{m}+Y_{m} Z_{m}\right)}
\end{gathered}
$$

Using invariance of the commutation relations by cyclic permutation of $X, Y, Z$ we also have $\left[Y_{n}, X_{m}^{2}+Y_{m}^{2}+Z_{m}^{2}\right]=\left[Z_{n}, X_{m}^{2}+Y_{m}^{2}+Z_{m}^{2}\right]=0$, and the result follows.

We deduce from this that the family of operators $\left(X_{n}^{2}+Y_{n}^{2}+Z_{n}^{2}\right) ; n \geq 1$ is commutative, and therefore defines a classical process. We shall compute its distribution now. For this we introduce the operators $S_{n}=\sqrt{X_{n}^{2}+Y_{n}^{2}+Z_{n}^{2}+I}$. By the preceding Lemma, the family $\left(S_{n}\right)_{n \geq 0}$ is a commuting family of operators, therefore one can consider their joint distribution.

Theorem 3.2. Let us take for $\omega$ the tracial state, then the operators $\left(S_{n}\right.$; $n \geq 1)$ form a Markov chain, with values in the positive integers, such that $P\left(S_{1}=2\right)=1$, and transition probabilities

$$
p(k, k+1)=\frac{k+1}{2 k} ; \quad p(k, k-1)=\frac{k-1}{2 k} .
$$


In order to prove the theorem, it is enough to consider the process up to a finite time $n$. So we shall restrict considerations to a finite product $M_{2}(\mathbb{C})^{\otimes n}$ acting on $\left(\mathbb{C}^{2}\right)^{\otimes n}$. We remark that the state $\omega^{\otimes n}$ on $\left(\mathbb{C}^{2}\right)^{\otimes n}$ is the unique tracial state, and it gives, to every orthogonal projection on a subspace $V$, the value $\frac{\operatorname{dim}(V)}{2^{n}}$. We shall find a basis of this space consisting of joint eigenvectors of the operators $\left(S_{k} ; 1 \leq k \leq n\right)$. For this we analyze the action of the operators $A_{n}^{+}:=\frac{1}{2}\left(X_{n}-i Y_{n}\right)$ and $A_{n}^{-}=\frac{1}{2}\left(X_{n}+i Y_{n}\right)$. One has

$$
A_{n}^{+}=\sum_{j=1}^{n} I^{\otimes(j-1)} \otimes \alpha^{+} \otimes I^{\otimes n-j} \quad A_{n}^{-}=\sum_{j=1}^{n} I^{\otimes(j-1)} \otimes \alpha^{-} \otimes I^{\otimes n-j}
$$

where

$$
\alpha^{+}=\left(\begin{array}{ll}
0 & 0 \\
1 & 0
\end{array}\right) \quad \alpha^{-}=\left(\begin{array}{ll}
0 & 1 \\
0 & 0
\end{array}\right) .
$$

Let us call $e_{0}, e_{1}$ the canonical basis of $\mathbb{C}^{2}$. An orthonormal basis of $\left(\mathbb{C}^{2}\right)^{\otimes n}$ is given by the vectors $e_{U} ; U \subset\{1,2, \ldots, n\}$ where $e_{U}=e_{i_{1}} \otimes \ldots \otimes e_{i_{n}}, i_{k}=1$ if $k \in U$ and $i_{k}=0$ if $k \notin U$. In terms of this basis the action of $A_{n}^{+}$and $A_{n}^{-}$ is given by

$$
\begin{aligned}
A_{n}^{+} e_{U} & =\sum_{k \notin U} e_{U \cup\{k\}} \\
A_{n}^{-} e_{U} & =\sum_{k \in U} e_{U \backslash\{k\}} \\
Z_{n} e_{U} & =(n-2|U|) e_{U}
\end{aligned}
$$

Let us consider the vector $e_{\emptyset}=e_{0}^{\otimes n}$ and its images by the powers of $A_{n}^{-}$, normalized to have norm one. These are the vectors

$$
\varepsilon_{n}^{j}=\sqrt{\frac{j !(n-j) !}{n !}} \sum_{U \subset\{1,2, \ldots, n\} ;|U|=j} e_{U} \quad j=0,1, \ldots, n
$$

and these vectors are orthogonal. The action of the operators $A_{n}^{+}, A_{n}^{-}, Z_{n}$ on these vectors is given by

$$
\begin{aligned}
A_{n}^{+} \varepsilon_{n}^{j} & =\sqrt{(j+1)(n-j)} \varepsilon_{n}^{j+1} \\
A_{n}^{-} \varepsilon_{n}^{j} & =\sqrt{j(n-j+1)} \varepsilon_{n}^{j-1} \\
Z_{n} \varepsilon_{n}^{j} & =(n-2 j) \varepsilon_{n}^{j} \\
S_{n} \varepsilon_{n}^{j} & =(n+1) \varepsilon_{n}^{j}
\end{aligned}
$$

In particular we see that these vectors belong to the eigenspace of $S_{n}$ of eigenvalue $n+1$. We shall generalize this computation to find the common eigenspaces of the operators $S_{1}, S_{2}, \ldots, S_{n}$.

Lemma 3.3. Let $J=\left(j_{1}, \ldots, j_{n}\right)$ be a sequence of integers such that

- i) $j_{1}=2$

- ii) $j_{i} \geq 1$ for all $i \leq n$

- iii) $\left|j_{i+1}-j_{i}\right|=1$ for all $i \leq n-1$ 
then there exists a subspace $H_{J}$ of $\left(\mathbb{C}^{2}\right)^{\otimes n}$ of dimension $j_{n}=l+1$, which is an eigenspace of $S_{1}, \ldots, S_{n}$, with respective eigenvalues $j_{1}, \ldots, j_{n}$, and an orthonormal basis $\left(\phi_{0}, \ldots, \phi_{l}\right)$ such that

$$
\begin{aligned}
A_{n}^{+} \phi_{j} & =\sqrt{(j+1)(l-j)} \phi_{j+1} \\
A_{n}^{-} \phi_{j} & =\sqrt{j(l-j+1)} \phi_{j-1} \\
Z_{n} \phi_{j} & =(l-2 j) \phi_{j}
\end{aligned}
$$

Furthermore, the spaces $H_{J}$ are orthogonal and $\left(\mathbb{C}^{2}\right)^{\otimes n}=\oplus_{J} H_{J}$.

Proof of the lemma. We shall use induction on $n$. The lemma is true for $n=1$, using $\phi_{0}=e_{0} ; \phi_{1}=e_{1}$. Assume the lemma holds for $n$, and let $J=\left(j_{1}, \ldots, j_{n}\right)$ be a sequence satisfying the conditions $\left.\left.\left.i\right), i i\right), i i i\right)$, of the lemma. We shall decompose the space $H_{J} \otimes \mathbb{C}^{2}$ as a direct sum of two subspaces. Let the vectors $\psi_{j}$ and $\eta_{j}$ be defined by

$$
\psi_{j}=\sqrt{\frac{l-j+1}{l+1}} \phi_{j} \otimes e_{0}+\sqrt{\frac{j}{l+1}} \phi_{j-1} \otimes e_{1} \quad j=0, \ldots, l+1
$$

and

$$
\eta_{j}=\sqrt{\frac{j+1}{l+1}} \phi_{j+1} \otimes e_{0}-\sqrt{\frac{l-j}{l+1}} \phi_{j} \otimes e_{1} \quad j=0, \ldots, l-1
$$

It is easy to check that these vectors form an orthonormal basis of the tensor product $H_{J} \otimes \mathbb{C}^{2}$, and a simple computation using

$$
X_{n+1}=X_{n}+I^{\otimes n} \otimes \sigma_{x}, Y_{n+1}=Y_{n}+I^{\otimes n} \otimes \sigma_{y}, Z_{n+1}=Z_{n}+I^{\otimes n} \otimes \sigma_{z}
$$

shows that these vectors have the right behaviour under these operators.

We can now prove theorem 3.2, indeed for any sequence satisfying the hypotheses of lemma 3.3 one has

$$
P\left(S_{1}=j_{1}, \ldots, S_{n}=j_{n}\right)=\frac{j_{n}}{2^{n}}=\frac{j_{1}}{2} \frac{j_{2}}{2 j_{1}} \ldots \frac{j_{n}}{2 j_{n-1}}
$$

where the right hand side is given by the distribution of the Markov chain of theorem 3.2.

We shall give another, more conceptual, proof of the former result in section 5, using the representation theory of the group $S U(2)$ and ClebschGordan formulas. For this we shall interpret the quantum Bernoulli random walk as a Markov process with values in a noncommutative space, but we need first to give some definitions pertaining to bialgebras and group algebras, which we do in the next section. 


\section{Bialgebras and Group Algebras}

\subsection{Coproducts}

Let $X$ be a finite set, and $\mathcal{F}(X)$ be the algebra of complex functions on $X$. A composition law on $X$ is a map $X \times X \rightarrow X$. This gives rise to a unit preserving algebra morphism $\Delta: \mathcal{F}(X) \rightarrow \mathcal{F}(X \times X)$, where $\mathcal{F}(X \times X)$ is the algebra of functions on $X \times X$, and one has a natural isomorphism $\mathcal{F}(X \times X) \sim \mathcal{F}(X) \otimes \mathcal{F}(X)$. Conversely, such an algebra morphism $\Delta$ : $\mathcal{F}(X) \rightarrow \mathcal{F}(X) \otimes \mathcal{F}(X)$ comes from a composition law, and many properties of the composition law can be read on it. For example, associativity translates into coassociativity of the coproduct which means that

$$
(\Delta \otimes I) \circ \Delta=(I \otimes \Delta) \circ \Delta
$$

whereas commutativity gives cocommutativity for the coproduct, which means that

$$
v \circ \Delta=\Delta
$$

where $v: \mathcal{F}(X) \otimes \mathcal{F}(X) \rightarrow \mathcal{F}(X) \otimes \mathcal{F}(X)$ is the flip automorphism $v(a \otimes$ $b)=b \otimes a$. In order to obtain an analogue of a composition law in the noncommutative context, one can define a coproduct for any algebra $A$ as a morphism $\Delta: A \rightarrow A \otimes A$, however in general the algebraic tensor product is too small for obtaining interesting examples. Think for example to the case $A=C_{b}(X), X$ a locally compact space, and see that $A \otimes A \subset C_{b}(X \times X)$ is a small subspace. We shall therefore consider coproducts which take values in a suitable completion of the algebraic tensor product. An algebra endowed with a coassociative coproduct is called a bialgebra. If the algebra is a $C^{*}$ (resp. a von Neumann) algebra and the tensor product is the minimal $C^{*}$ product (resp. the von Neumann algebra tensor product), then one has a $C^{*}$ (resp. a von Neumann) bialgebra.

Some further properties of a coproduct are the existence of the dual notion of the unit element and the inverse, which are respectively called a counit, $\varepsilon: A \rightarrow \mathbb{C}$ and an antipode, $i: \Delta \rightarrow \Delta$.

A Hopf algebra is a bialgebra with a unit and an antipode, satisfying some compatibility conditions. I refer for example to $[\mathrm{K}]$ for an exposition of Hopf algebras and quantum groups.

\subsection{Some Algebras Associated to a Compact Group}

We shall investigate in more details the notions above in the case of the group algebra of a compact group, which we assume for simplicity to be separable. Recall that every representation of a compact group can be made unitary, and can be reduced to an orthogonal direct sum of irreducible representations. The 
right regular representation of a compact group $G$ is the representation of $G$ on $L^{2}(G, m)$ (where $m$ is a Haar measure on $G$ ) by right translations $\rho_{g} f(h)=$ $f\left(h g^{-1}\right)$ and the left regular representation acts by left translations $\lambda_{g} f(h)=$ $f(g h)$. A fundamental theorem is the Peter-Weyl theorem. It states that every irreducible representation of $G$ arises in the decomposition of the left (or right) regular representation, actually one has an orthogonal direct sum

$$
L^{2}(G)=\oplus_{\chi \in \hat{G}} E_{\chi}
$$

where $\hat{G}$ is the (countable) set of equivalence classes of irreducible representations of $G$, and for $\chi \in \hat{G}, E_{\chi}$ is the space of coefficients of the representation i.e. the vector space generated by functions on $G$ of the form

$$
f(g)=\langle\chi(g) u, v\rangle
$$

where $u, v$ are vectors in the representation space of $\chi(\langle.,$.$\rangle being an invariant$ hermitian product on the space). The space $E_{\chi}$ is finite dimensional, its dimension being $\operatorname{dim}(\chi)^{2}$, and it is an algebra for the convolution product on $G$, isomorphic to the matrix algebra $M_{n}(\mathbb{C})$ with $n=\operatorname{dim}(\chi)$. We shall denote this space $M_{\chi}$ when we want to emphasize its algebra structure.

We shall describe several algebras associated to $G$. The first one is the convolution algebra $\mathcal{A}^{0}(G)$ generated by the coefficients of the finite dimensional representations. As a vector space it is the algebraic direct sum $\mathcal{A}^{0}(G)=\oplus_{\chi} M_{\chi} \sim \oplus_{\chi} M_{\operatorname{dim}(\chi)}(\mathbb{C})$. There are larger algebras such as $L^{1}(G)$ the space of integrable functions (with respect to the Haar measure on $G$ ), and $C^{*}(G)$ the $C^{*}$ algebra generated by $L^{1}(G)$. This algebra consists of sequences $\left(m_{\chi} ; \chi \in \hat{G}\right)$ such that $m_{\chi} \in M_{\chi} ;\left|m_{\chi}\right| \rightarrow 0$ as $\chi \rightarrow \infty$.

The multiplier algebra of $C^{*}(G)$ coincides with the von Neumann algebra $\mathcal{A}(G)$, which is generated (topologically) by the left translation operators $\lambda_{g} ; g \in G$, it consists of sequences $\left(m_{\chi} ; \chi \in \hat{G}\right)$ such that $\sup _{\chi}\left|m_{\chi}\right|<\infty$. In both cases, the norm in these algebras is $\sup _{\chi}\left|m_{\chi}\right|$. Note that the left and right translation operators $\lambda_{g}$ and $\rho_{g}$ are unitary, and the right translation operators generate the commutant of $\mathcal{A}(G)$.

When the group $G$ is abelian, there is a natural isomorphism, given by Fourier transform, between the algebra $\mathcal{A}(G)$ and $L^{\infty}(\hat{G})$ where here $\hat{G}$ is the group of characters of $G$ (this is consistent with our earlier notation since then all irreducible representations of $G$ are one dimensional and are thus characters). This group of characters is a discrete abelian group, and its Haar measure is the counting measure.

In the general case, the algebra $L^{\infty}(\hat{G})$ is isomorphic with the center of $\mathcal{A}(G)$, since a bounded function on $\hat{G}$ can be identified with a sequence $\left(m_{\chi}\right)_{\chi \in \hat{G}}$ of scalar operators.

Any closed subgroup of $G$ generates a von Neumann subalgebra $\mathcal{A}(H)$ furthermore the coproduct $\Delta$ restricts to this subalgebra and defines a coproduct $\Delta: \mathcal{A}(H) \rightarrow A(H) \otimes A(H)$. If the subgroup is abelian, then this subalgebra is commutative and is isomorphic to the algebra $L^{\infty}(\hat{H})$, 
Finally we shall also use the algebra $\hat{\mathcal{A}}(G)=\prod_{\chi} M_{\chi}$ which consists in unbounded operators on $L^{2}(G)$, with common dense domain $\oplus_{\chi} E_{\chi}$ (algebraic direct sum), affiliated with the von Neumann algebra $\mathcal{A}(G)$. One has natural inclusions

$$
\mathcal{A}^{0}(G) \subset C^{*}(G) \subset M\left(C^{*}(G)\right)=\mathcal{A}(G) \subset \hat{\mathcal{A}}(G)
$$

The algebra $\hat{\mathcal{A}}(G) \otimes \hat{\mathcal{A}}(G)$ is an algebra of operators on the algebraic direct sum $\oplus_{\chi, \chi^{\prime}} E_{\chi} \otimes E_{\chi^{\prime}}$ and we denote by $\hat{\mathcal{A}}(G) \hat{\otimes} \hat{\mathcal{A}}(G)$ its completion for simple convergence on $\oplus_{\chi, \chi^{\prime}} E_{\chi} \otimes E_{\chi^{\prime}}$. One has

$$
\hat{\mathcal{A}}(G) \otimes \hat{\mathcal{A}}(G)=\prod_{\chi} M_{\chi} \otimes \prod_{\chi} M_{\chi} \subset \hat{\mathcal{A}}(G) \hat{\otimes} \hat{\mathcal{A}}(G) \sim \prod_{\chi, \chi^{\prime}} M_{\chi} \otimes M_{\chi^{\prime}}
$$

The $*$ algebra structure extends obviously to $\hat{\mathcal{A}}(G)$, and an element is positive if and only if its components are positive.

\subsection{The Coproduct}

The coproduct formula $\Delta: \lambda_{g} \rightarrow \lambda_{g} \otimes \lambda_{g}$ extends by linearity and continuity to the von Neumann algebra $\mathcal{A}(G)$ if we use the von Neumann algebra tensor product. It defines a structure of cocommutative von Neumann bialgebra on $\mathcal{A}(G)$. One can also define an extension of the coproduct

$$
\hat{\Delta}: \hat{\mathcal{A}}(G) \rightarrow \hat{\mathcal{A}}(G) \hat{\otimes} \hat{\mathcal{A}}(G) .
$$

Indeed it is easy to check that for any $\chi \in \hat{G}$, and $a \in M_{\chi}$, the operator $\Delta(a)$ is nonzero on the space $E_{\chi^{\prime}} \otimes E_{\chi^{\prime \prime}}$ if and only if $\chi$ has a non zero multiplicity in the decomposition of the tensor product representation $E_{\chi^{\prime}} \otimes E_{\chi^{\prime \prime}}$. It follows that for any sequence $\left(a_{\chi}\right)_{\chi \in \hat{G}} \in \prod_{\chi \in \hat{G}} M_{\chi}$ the sum $\sum_{\chi \in \hat{G}} \Delta\left(a_{\chi}\right)$ is a finite sum in each component $E_{\chi} \otimes E_{\chi^{\prime}}$ therefore it defines an element in $\hat{\mathcal{A}}(G) \hat{\otimes} \hat{\mathcal{A}}(G) \sim \prod_{\chi, \chi^{\prime}} M_{\chi} \otimes M_{\chi^{\prime}}$.

One can define the convolution of two finite weights $\mu$ and $\nu$ by the formula

$$
\mu * \nu=(\mu \otimes \nu) \circ \Delta
$$

since the coproduct is cocommutative and coassociative, one checks that this is an associative and commutative operation.

\subsection{The Case of Lie Groups}

For compact Lie groups there is another algebra of interest, which is the envelopping algebra of the Lie algebra. Recall that the Lie algebra of a Lie 
group is composed of right invariant vector fields on the group. As such it acts on the $L^{2}$ space as a family of unbounded operators. Therefore the Lie algebra is a subspace of the "big" algebra $\hat{\mathcal{A}}(G)$, and the algebra generated by this subspace is naturally isomorphic to the envelopping Lie algebra (for simply connected groups). The elements of the Lie algebra have the following behaviour with respect to the extended coproduct on $\hat{\mathcal{A}}(G)$

$$
\Delta(X)=X \otimes I+I \otimes X
$$

This can be seen by taking derivatives with respect to $s$ in the equation

$$
\Delta\left(e^{s X}\right)=e^{s X} \otimes e^{s X}
$$

where $e^{s X} ; s \in \mathbb{R}$ is the one parameter subgroup generated by $X$.

\subsection{States and Weights}

A normal state $\nu$ on $\mathcal{A}(G)$ is determined by its value on the generators $\lambda_{g}$, thus by the function $\phi_{\nu}(g)=\nu\left(\lambda_{g}\right)$. It is a classical result that a function $\phi$ on $G$ corresponds to a state on $C^{*}(G)$, and to a normal state on $\mathcal{A}(G)$, if and only if it is a continuous positive definite function on the group, satisfying $\phi(e)=1$.

Every normal weight on $\mathcal{A}(G)$ is given by a sequence of weights $\left(\nu_{\chi}\right)_{\chi \in \hat{G}}$ on each of the subalgebras $M_{\chi}$, therefore for a weight $\nu$ on $\mathcal{A}(G)$ there exists a sequence of positive elements $f_{\chi} \in M_{\chi}$ such that $\nu_{\chi}(a)=\operatorname{Tr}\left(a f_{\chi}\right)$ for all $a \in M_{\chi}$. Conversely any such sequence $f_{\chi}$ defines a normal weight, thus normal weights on $\mathcal{A}(G)$ correspond to positive elements in $\hat{\mathcal{A}}(G)$.

\section{Random Walk on the Dual of $S U(2)$}

\subsection{The Dual of $S U(2)$ as a Noncommutative Space}

We shall now interpret the process constructed in section 3 as a random walk on a noncommutative space. For this we consider the group $S U(2)$ of unitary $2 \times 2$ matrices with determinant 1 . It is well known that the irreducible representations of this group are finite dimensional, moreover, for each integer $n \geq 1$ there exists, up to equivalence, exactly one irreducible representation of dimension $n$, therefore one has

$$
\mathcal{A}(S U(2))=\oplus_{n=1}^{\infty} M_{n}(\mathbb{C})
$$


as a von Neumann algebra direct sum and similarly the $C^{*}$ algebra of $S U(2)$ can be identified with the algebra of sequences $\left(M_{n}\right)_{n \geq 1}$ where $M_{n}$ is an $n \times n$ matrix and one has $\left\|M_{n}\right\| \rightarrow 0$ as $n \rightarrow \infty$. We shall interpret this $C^{*}$-algebra as the space of "functions" vanishing at infinity on some noncommutative space. The associated von Neumann algebra corresponds to the space of "bounded functions" on our noncommutative space. In order to get a better picture of this space it is desirable to have a geometric understanding of its structure. For this we first note that this space has a continuous group of symmetries, since the group $S U(2)$ acts on the algebra by inner automorphisms. Since the elements $\pm I$ act trivially, this is really an action of the quotient $S U(2) /\{ \pm I\}$ which is isomorphic with the group $S O(3)$, therefore this space has a three dimensional rotational symmetry. We can understand this symmetry by looking at some special elements in the larger algebra $\hat{\mathcal{A}}(S U(2))$, which correspond to "unbounded functions". Let us consider the self-adjoint elements corresponding to the Pauli matrices, viewed as elements of the Lie algebra of $S U(2)$ (or rather the complexified Lie algebra). These define unbounded operators on $L^{2}(S U(2))$, which we shall denote $X, Y, Z$, and which lie in $\hat{\mathcal{A}}(S U(2))$. A good way to think about these three functions is as three "coordinates" on our space, corresponding to three orthogonal directions. Each of these elements has a spectrum which is exactly the set of integers (which you can view as the group dual to the one parameter group generated by one of these Lie algebra elements). Moreover this is true also of any linear combination $x X+y Y+z Z$ with $x^{2}+y^{2}+z^{2}=1$. This means that if you are in this space and try to measure your position, you can measure, as in quantum mechanics, one coordinate in some direction $(x, y, z)$ using the operator $x X+y Y+z Z$, and you will always find an integer. Thus the space has some discrete feature, in that you always get integer numbers for your coordinates, but also a continuous rotational symmetry which comes from the action of $S U(2)$ by automorphisms of the algebra. This is obviously impossible to obtain in a classical space. Of course since the operators in different directions do not commute, you cannot measure your position in different directions of space simultaneously. What you can do nevertheless is measure simultaneoulsy one coordinate in space, and your distance to the origin. This last measurement is done using the operator $D=\sqrt{I+X^{2}+Y^{2}+Z^{2}}-I$ which is in the center of the algebra $\hat{\mathcal{A}}(S U(2))$, and therefore can be measured simultaneously with any other operator. Its eigenvalues are the nonnegative integers $0,1,2 \ldots$, and its spectral projections are the identity elements of the algebras $M_{n}(\mathbb{C})$, more precisely, one has in $\hat{\mathcal{A}}(S U(2))$

$$
D=\sum_{n=1}^{\infty}(n-1) I_{M_{n}(\mathbb{C})}
$$

We thus see that the subalgebra $M_{n}(\mathbb{C})$ is a kind of "noncommutative sphere of radius $n-1$ ", and moreover by looking at the eigenvalues of the operators $x X+y Y+z Z$ in the corresponding representation, we see 


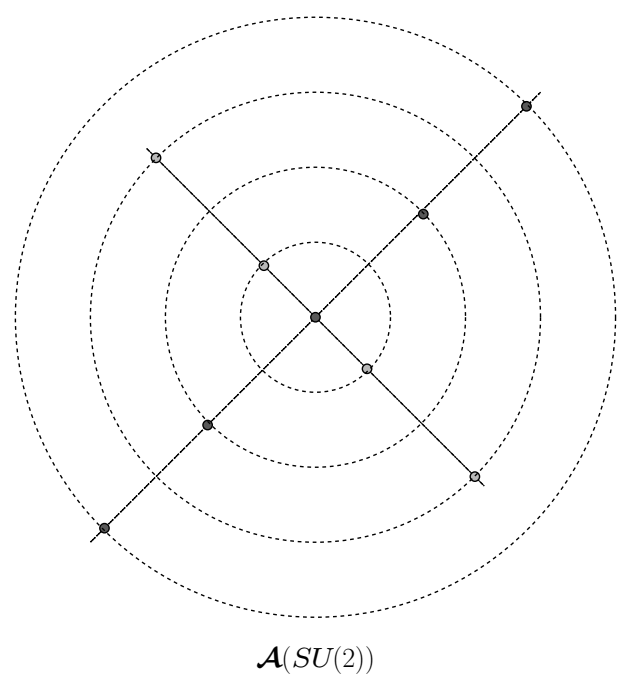

Fig. 1 Noncommutative space underlying $\mathcal{A}(S U(2))$.

that on any "radius" of this sphere, corresponding to a direction of space, the coordinate on this radius can only take the $n$ values $n-1, n-3$, $n-5, \ldots,-n+1$.

If we rescale the noncommutative sphere of large radius to have radius 1, it looks more and more like a classical sphere, see e.g. [Rie] for a precise statement.

\subsection{Construction of the Random Walk}

Let $\omega$ be a state on $M_{2}(\mathbb{C})$, which we can also consider as a state on $\mathcal{A}(S U(2))$ by the projection $\mathcal{A}(S U(2)) \rightarrow M_{2}(\mathbb{C})$. Let us consider the infinite tensor product algebra, with respect to the product state $\nu=\omega^{\otimes \infty}$ on $\left.\mathcal{N}=\otimes_{1}^{\infty} \mathcal{A}(S U(2))\right)$.

Let $T: \mathcal{N} \rightarrow \mathcal{N}$ be defined by $\Delta \otimes s$ where $s: \mathcal{A}(S U(2))^{[2, \infty[} \rightarrow$ $\mathcal{A}(S U(2))^{[3, \infty}\left[\right.$ is the obvious shift isomorphism. Let $j_{n}: \mathcal{A}(S U(2)) \rightarrow \mathcal{N}$ be the morphisms defined by by $j_{n}=T^{n} \circ i$ where $i(a)=a \otimes I^{\infty}$ is the GNS representation of $\mathcal{A}(S U(2))$ associated with $\omega$, acting on the first factor. Note that one has actually $\mathcal{N}=\otimes_{1}^{\infty} M_{2}(\mathbb{C})$ and $i(a)=\rho_{2}(a) \otimes I^{\infty}$ where $\rho_{2}$ is a two dimensional irreducible representation. The morphisms $j_{n}$ can be extended to the large algebra $\hat{\mathcal{A}}(S U(2))$ and for $V$ in the Lie algebra, using the formula for the coproduct one checks that

$$
j_{n}(V)=\sum_{i=1}^{n} I^{\otimes(i-1)} \otimes V \otimes I^{\otimes \infty}
$$


Thus the quantum Bernoulli random walk is obtained by taking the operators $\left(j_{n}(X), j_{n}(Y), j_{n}(Z)\right)$ where $X, Y, Z$ are the Lie algebra elements corresponding to the Pauli matrices. For $n \geq 0$, we let $\mathcal{N}_{n}$ be the algebra generated by the first $n$ factors in the tensor product. There exists a conditional expectation $\nu\left(. \mid \mathcal{N}_{n}\right)$ with respect to the state $\nu$ onto such a subalgebra, it is given simply by $I \otimes \omega^{\infty}$ on the factorization $\mathcal{N}=\mathcal{A}(S U(2))^{\otimes n} \otimes \mathcal{A}(S U(2))^{\otimes[n+1, \infty[}$.

The family of morphisms $\left(j_{n}\right)_{n \geq 1}$ form a noncommutative process wih values in the dual of $S U(2)$. The following proposition is left to the reader, as an exercise in manipulation of coproducts.

Proposition 5.1. The family of morphisms $j_{n} ; n \geq 1$, together with the family of algebras $\mathcal{N}, \mathcal{N}_{n} ; n \geq 1$, and the conditional expectations $\nu\left(. \mid \mathcal{N}_{n}\right)$, form a dilation of the completely positive map $\mathcal{A}(S U(2)) \rightarrow \mathcal{A}(S U(2))$ given by $\Phi_{\omega}=(I \otimes \omega) \circ \Delta$.

Let us now consider the three one-parameter subgroups generated by the Pauli matrices, they consist respectively of the matrices

$$
\left(\begin{array}{cc}
\cos \theta & i \sin \theta \\
i \sin \theta & \cos \theta
\end{array}\right) \quad\left(\begin{array}{cc}
\cos \theta & \sin \theta \\
-\sin \theta & \cos \theta
\end{array}\right) \quad\left(\begin{array}{cc}
e^{i \theta} & 0 \\
0 & e^{-i \theta}
\end{array}\right) \quad \theta \in[0,2 \pi[
$$

Each of these subgroups generates a commutative von Neumann subalgebra of $\mathcal{A}(S U(2))$, which is isomorphic with the group von Neumann algebra of the group $U(1)$ of complex numbers of modulus 1 . Such a von Neumann algebra is isomorphic, by Pontryagin duality, to the algebra of bounded functions on the dual group, therefore the restriction of the dilation to this subalgebra provides a random walk on this dual group, which is isomorphic to $\mathbb{Z}$. Thus we recover, from the abstract considerations on duals of compact groups, our concrete Bernoulli random walks. In terms of our noncommutative space, we can observe a particle undergoing this random walk along any fixed direction of space, and what we see is a Bernoulli random walk (recall that the coordinate in some fixed direction of space can only take integer values).

We now turn to the spin process which can be interpreted in terms of the restriction of the dilation $\left(j_{n}\right)_{n \geq 1}$ to the center of the group algebra $\mathcal{A}(G)$. This center consists of operators of the form $\left(m_{\chi} ; \chi \in \hat{G}\right)$ where each $m_{\chi}$ is a scalar operator in $M_{\chi}$, it is a commutative algebra, isomorphic with the algebra of bounded functions on $\hat{G}$ (recall that we have assumed that $\hat{G}$ is countable). Equivalently it is the algebra generated by the operator $D$ i.e. the algebra of operators of the form $f(D)$ where $f$ is a bounded complex function on the nonnegative integers. It is also easy to compute the restriction of the completely positive map $\Phi_{\omega}$ to this center. It is given by the Clebsch-Gordan formula which computes the decomposition into irreducible of a tensor product of representations of the group $S U(2)$. What we need is the formula (where $\rho_{n}$ is the $n$-dimensional irreducible representation)

$$
\rho_{2} \otimes \rho_{n}=\rho_{n-1} \oplus \rho_{n+1}
$$


which tells us that the Markov chain can jump from $n$ to either $n-1$ or $n+1$, furthermore the transition probabilities are proportional to the dimensions of the targets, since we are in the trace state. We see that the restriction of $j_{n}$ to this algebra thus corresponds to the spin process.

Finally there are other commutative algebras which are invariant under the completely positive map. They are generated by the center of $\mathcal{A}(S U(2))$ and by a one parameter subgroup. Each such algebra is a maximal abelian subalgebra of $\mathcal{A}(S U(2))$, and its spectrum can be identified with the set of pairs $(m, n)$ of integers such that $n \geq 1$ and $m \in\{n-1, n-3, \ldots-n+1\}$. One can compute the associated Markov semigroup, using the Clebsch Gordan formulas for products of coefficient functions of the group $S U(2)$. However the images of such an algebra by the morphisms $\left(j_{n}\right)_{n \geq 1}$ do not commute. Thus in this way we get a noncommutative dilation of a purely commutative semigroup. We will come back to this Markov chain when we study Pitman's theorem and quantum groups.

\section{Random Walks on Duals of Compact Groups}

It is easy to generalize the construction of the preceding section by replacing the group $S U(2)$ by an arbitrary compact group $G$. We will do a construction parallel to the usual construction of a random walk on a group. Let $\phi_{0}$ and $\phi$ be continuous positive definite functions on $G$, with $\phi_{0}(e)=\phi(e)=1$, thus these functions correspond to normal states $\nu_{0}$ and $\nu$ on $\mathcal{A}(G)$. The state $\nu_{0}$ will play the role of initial condition of our Markov chain, whereas the state $\nu$ represents the distribution of the increments. To the state $\nu$ is associated a completely positive map

$$
\Phi_{\nu}: \mathcal{A}(G) \rightarrow \mathcal{A}(G) \quad \Phi_{\nu}=(I \otimes \nu) \circ \Delta .
$$

The completely positive map generates a semigroup $\Phi_{\nu}^{n} ; n \geq 1$. We now build the infinite tensor product $\mathcal{N}=\mathcal{A}(G)^{\infty}$ with respect to the state $\nu_{0} \otimes \nu^{\otimes \infty}$, and obtain a noncommutative probability space $(\mathcal{N}, \omega)$. Let $T: \mathcal{N} \rightarrow \mathcal{N}$ defined by $\Delta \otimes s$ where $s: \mathcal{A}(G)^{[1, \infty[} \rightarrow \mathcal{A}(G)^{[2, \infty[}$ is the obvious isomorphism. Let $j_{n}: \mathcal{A}(G) \rightarrow \mathcal{N}$ be the morphisms defined by induction $j_{n}=T^{n} \circ i$ where $i(a)=a \otimes I^{\infty}$ is the inclusion of $\mathcal{A}(G)$ into the first factor (strictly speaking this is an inclusion only if the state $\nu_{0}$ is faithful).

Let us translate the above construction in the case of an abelian group, in terms of the dual group $\hat{G}$. The states $\nu$ and $\nu_{0}$ correspond to probability measures on $\hat{G}$, the probability space is now the product of an infinite number of copies of $\hat{G}$, with the product probability $\nu_{0} \otimes \nu^{\otimes \infty}$, and the maps $j_{n}$ correspond to functions $X_{n}: \hat{G}^{\infty} \rightarrow G$ given by $X_{n}\left(g_{0}, g_{1}, \ldots, g_{k}, \ldots\right)=$ $g_{0} g_{1} \ldots g_{n-1}$. We thus recover the usual construction of a random walk. 
For $n \geq 0$, we let $\mathcal{N}_{n}$ be the algebra generated by the first $n+1$ factors in the tensor product. There exists a conditional expectation $\omega\left(. \mid \mathcal{N}_{n}\right)$ with respect to the state $\omega$ onto such a subalgebra, it is given simply by $I \otimes \nu^{\infty}$ on the factorization $\mathcal{N}=\mathcal{A}(G)^{\otimes(n+1)} \otimes \mathcal{A}(G)^{\otimes \infty}$.

Proposition 6.1. The morphisms $\left(j_{n}\right)_{n \geq 0}$, together with the von Neumann algebras $\mathcal{N}, \mathcal{N}_{n}, n \geq 0$ and the state $\omega$, form a dilation of the completely positive semigroup $\left(\Phi_{\nu}^{n}\right)_{n \geq 1}$, with initial distribution $\nu_{0}$.

The proof of this proposition follows exactly the case of $S U(2)$ treated in the preceding section.

Let $H$ be a closed commutative subgroup of $G$, then its dual group $\hat{H}$ is a countable discrete abelian group. The von Neumann subalgebra $\mathcal{A}(H)$ generated by $H$ in $\mathcal{A}(G)$ is isomorphic to $L^{\infty}(\hat{H})$, and the restriction of the positive definite function $\phi$ to $H$ is the Fourier transform of a probability measure $\mu$ on $\hat{H}$. The coproduct $\Delta$ restricts to a coproduct on the subalgebra generated by $H$, thus the images of $\mathcal{A}(H)$ by the morphisms $j_{n}$ generate commuting subalgebras of $\mathcal{N}$. These restrictions thus give a random walk on the dual group $\hat{H}$, whose independent increments are distributed according to $\mu$.

We now consider another commutative algebra, namely the center $\mathcal{Z}(G)$ of $\mathcal{A}(G)$. Recall that this center is isomorphic with the space $L^{\infty}(\hat{G})$ of bounded functions on the set of equivalence classes of irreducible representations of $G$. As a von Neumann algebra of operators on $L^{2}(G)$, it is generated by the convolution operators by integrable central functions (recall that a function $f$ on $G$ is central if it satisfies $f\left(g h g^{-1}\right)=f(h)$ for all $\left.h, g \in G\right)$.

Proposition 6.2. The algebras $j_{n}(\mathcal{Z}(G)) ; n \geq 1$ commute.

Proof. Let $a, b \in \mathcal{Z}(G)$ and let $k \leq l$ we have to prove that $j_{k}(a)$ and $j_{l}(b)$ commute. Let $a^{\prime}=i(a), b^{\prime}=i(b)$, and note that $a^{\prime}$ and $b^{\prime}$ belong to the center of $\mathcal{N}$. One has

$$
\begin{aligned}
j_{k}(a) j_{l}(b) & =T^{k}\left(a^{\prime}\right) T^{k}\left(T^{l-k}\left(b^{\prime}\right)\right) \\
& =T^{k}\left(a^{\prime} T^{l-k}\left(b^{\prime}\right)\right) \\
& =T^{k}\left(T^{l-k}\left(b^{\prime}\right) a^{\prime}\right) \\
& =T^{l}\left(b^{\prime}\right) T^{k}\left(a^{\prime}\right) \\
& =j_{l}(b) j_{k}(a)
\end{aligned}
$$

Furthermore one has.

Proposition 6.3. If the function $\phi$ is central then $\Phi_{\nu}(\mathcal{Z}(G)) \subset \mathcal{Z}(G)$.

Proof. Indeed if $\psi$ is central function, belonging to $\mathcal{A}^{0}(G)$, then $\Phi_{\nu}(\psi)=\phi \psi$ is a central function on the group, and thus defines an element of $\mathcal{Z}(G)$.

We deduce from the preceding propositions that, in the case when the increments correspond to a central state, the restriction of the dilation $\left(j_{n}\right)_{n \geq 0}$ to the center of the algebra $\mathcal{A}(G)$ defines a Markov process on $\hat{G}$, whose 
transition operator is given by the restriction of $\Phi_{\nu}$ to the center $\mathcal{Z}(G)$. We shall now give a more concrete form of the transition probabilites of this Markov chain.

Proposition 6.4. For $\chi$ an irreducible character of $G$, let

$$
\phi \chi=\sum_{\chi^{\prime} \in \hat{G}} h_{\phi}\left(\chi^{\prime}, \chi\right) \chi^{\prime}
$$

be the expansion of the positive definite central function $\phi \chi$ into a combination characters, then the probability transitions of the Markov chain obtained from the restriction of $\left(j_{n}\right)_{n \geq 1}$ to the center are given by

$$
p_{\phi}\left(\chi^{\prime}, \chi\right)=\frac{\operatorname{dim}(\chi)}{\operatorname{dim}\left(\chi^{\prime}\right)} h_{\phi}\left(\chi^{\prime}, \chi\right)
$$

Proof. For $\chi \in \hat{G}$, the convolution operator associated with the function $\operatorname{dim}(\chi) \chi$ is the minimal projection of $\mathcal{Z}(G)$ associated with $\chi$. In other words it corresponds to the indicator function $1_{\chi}$ in the isomorphism of $L^{\infty}(\hat{G})$ with $\mathcal{Z}(G)$. The transition operator for the restriction of the dilation to the center is the restriction to this center of $\Phi_{\omega}$. On the other hand, the transition probabilities $p_{\phi}\left(\chi, \chi^{\prime}\right)$ are related to these indicator functions by $\Phi_{\omega}\left(1_{\chi^{\prime}}\right)=$ $\sum_{\chi} p_{\phi}\left(\chi^{\prime}, \chi\right) 1_{\chi}$. The conclusion follows immediately.

\section{The Case of $S U(n)$}

\subsection{Some Facts About the Group SU(n)}

We shall investigate the quantum random walk defined in the preceding section when the group $G$ is the group $S U(n)$ of unitary matrices with determinant 1 . First we recall some basic facts about this group and its representations. Let $\mathbf{T} \subset S U(n)$ be the subgroup of diagonal matrices, which is a maximal torus. The group of characters of $\mathbf{T}$ is an $n-1$ dimensional lattice, generated by the elements (the notation $e$ is here to suggest the exponential function)

$$
e\left(e_{i}\right)\left(\left(\begin{array}{ccc}
u_{1} & & 0 \\
& \ddots & \\
0 & & u_{n}
\end{array}\right)\right)=u_{j}
$$

These elements satisfy the relation (written in additive notation) $e_{1}+$ $e_{2}+\ldots+e_{n}=0$, which corresponds to the relation $e\left(e_{1}\right) e\left(e_{2}\right) \ldots e\left(e_{n}\right)=1$ for the characters. We denote by $\mathbf{P}$ this group, and by $\mathbf{P}_{+}$the subset of positive weights, i.e.

$$
\mathbf{P}_{+}=\left\{m_{1} e_{1}+\ldots+m_{n-1} e_{n-1} \mid m_{1} \geq m_{2} \geq \ldots \geq m_{n-1}\right\}
$$




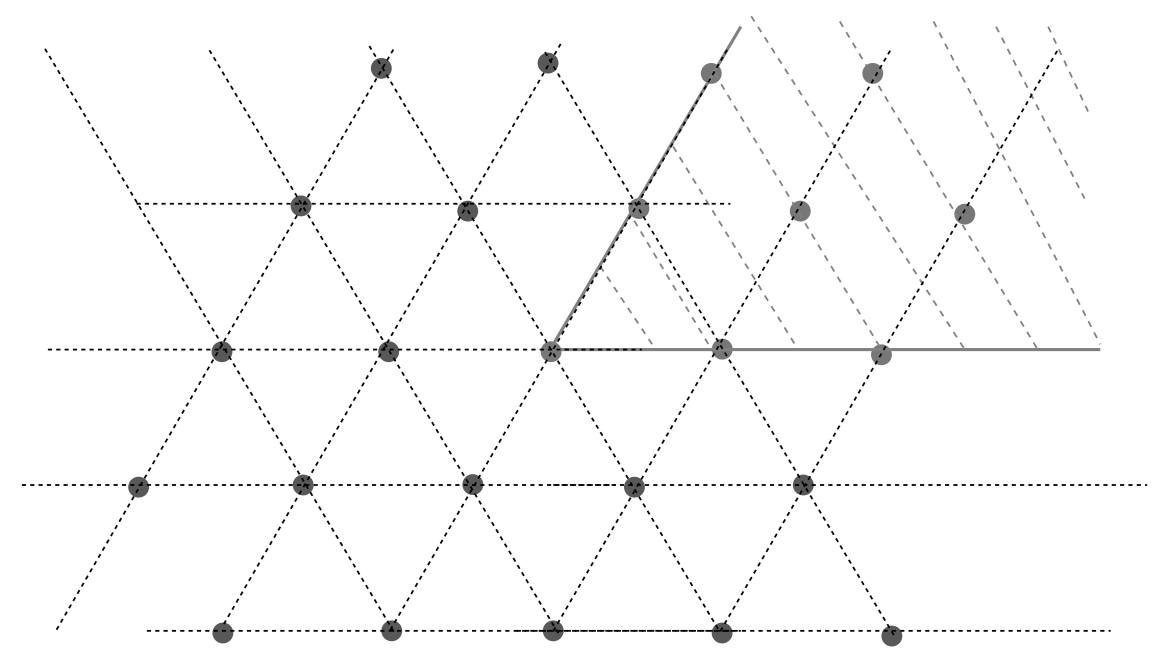

Fig. 2

We shall also need the set

$$
\mathbf{P}_{++}=\left\{m_{1} e_{1}+\ldots+m_{n-1} e_{n-1} \mid m_{1}>m_{2}>\ldots>m_{n-1}\right\}
$$

and note that the two sets are in bijection by

$$
\mathbf{P}_{++}=\mathbf{P}_{+}+\rho
$$

where $\rho=(n-1) e_{1}+(n-2) e_{2}+\ldots+e_{n-1}$ is the half sum of positive roots. The symmetric group acts on this character group by permutation of the $e_{i}$.

Below is a picture of $\mathbf{P}$ and $\mathbf{P}_{+}$for the group $S U(3)$. Thus $\mathbf{P}$ consists of the points in a triangular lattice in the plane, and $\mathbf{P}_{+}$is the intersection of this triangular lattice with a cone, fundamental domain for the action of the symmetric group $S_{3}$. The subset $\mathbf{P}_{++}$consists in points of $\mathbf{P}_{+}$which are in the interior of the cone.

Recall from the representation theory of the group $S U(n)$ (see e.g. [BtD], $[\mathrm{GW}]$, or $[\mathrm{Z}])$ that the equivalence classes of irreducible representations of $S U(n)$ are in one to one correspondence with the elements of $\mathbf{P}_{+}$, which are called "highest weights". For each $x \in \mathbf{P}$ let $e(x)$ be the associated character of $\mathbf{T}$, then the character of the representation with highest weight $x \in \mathbf{P}_{+}$ given, for $u \in \mathbf{T}$, by Weyl's character formula

$$
\chi_{x}(u)=\frac{\sum_{\sigma \in S_{n}} \epsilon(\sigma) e(\sigma(x+\rho))(u)}{\sum_{\sigma \in S_{n}} \epsilon(\sigma) e(\sigma(\rho))(u)}
$$

In particular the defining representation of $S U(n)$ has character $e\left(e_{1}\right)+\ldots+$ $e\left(e_{n}\right)$ corresponding to the highest weight $e_{1}$. The normalized positive definite 


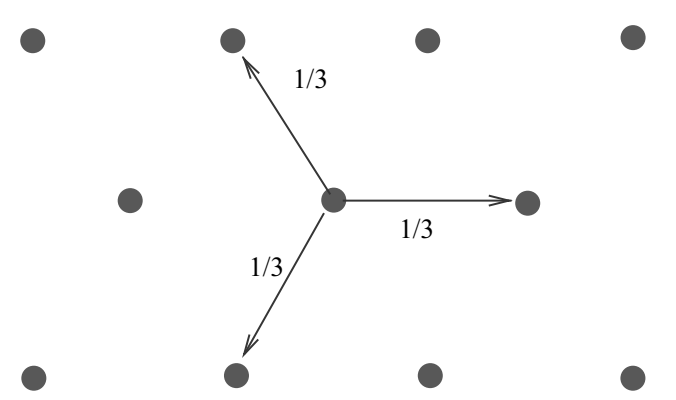

Fig. 3 The random walk on the dual of the maximal torus for SU(3).

function on $S U(n)$ corresponding to this character is $\phi(g)=\frac{1}{n} \operatorname{Tr}(g)$. We shall investigate the quantum random walk associated with this positive definite function.

\subsection{Two Classical Markov Chains}

We shall obtain two classical Markov chains by restricting the Markov chain associated with $\phi$ to suitable subalgebras of $C^{*}(S U(n))$. The first subalgebra is that generated by the maximal torus $\mathbf{T}$. This algebra is isomorphic to the algebra of functions vanishing at infinity on the dual group $\mathbf{P}$. It is easy to see that the restriction to the torus of the quantum random walk $\left(j_{n}\right)_{n \geq 0}$, constructed using $\phi$, is a random walk on the lattice $\mathbf{P}$ with increments distributed as $\frac{1}{n}\left(\delta_{e_{1}}+\ldots+\delta_{e_{n}}\right)$. Its one step transition probabilities are given by

$$
\begin{array}{ll}
p_{1}(x, y)=\frac{1}{n} & \text { if } y \in\left\{x+e_{1}, \ldots, x+e_{n}\right\} \\
p_{1}(x, y)=0 & \text { if not }
\end{array}
$$

We give the picture for the case of the group $S U(3)$.

The second subalgebra is the center of $C^{*}(S U(n))$. By the preceding section, it can be identified with the space of functions vanishing at infinity on $\mathbf{P}_{+}$. We shall rather use the identification of the set of irreducible representations with $\mathbf{P}_{++}$given by (7.1). As we saw in section 6 the restriction of the dilation $\left(j_{n}\right)_{n \geq 0}$ gives a classical Markov chain on the spectrum of the center, therefore we obtain in this way a Markov chain on $\mathbf{P}_{++}$, the generator of the Markov chain being given by the restriction of the generator of the quantum Markov chain on $\mathcal{A}(S U(2))$. 
We shall now derive a relation between these two Markov chains (the one obtained from the maximal torus, and the one from the center). The set of highest weights, $\mathbf{P}_{++}$is the intersection of $\mathbf{P}$ with the Weyl chamber, which is the cone

$$
\mathbf{C}=\left\{x_{1} e_{1}+\ldots+x_{n-1} e_{n-1} \mid x_{1}>x_{2}>\ldots>x_{n-1}\right\}
$$

We consider now the random walk on $\mathbf{P}$ killed at the exit of this cone. Thus the transition probabilites of this killed random walk are given by

$$
\begin{array}{ll}
p^{0}(x, y)=\frac{1}{n} & \text { if } y \in \mathbf{P}_{++} \cap\left\{x+e_{1}, \ldots, x+e_{n}\right\} \\
p^{0}(x, y)=0 & \text { if not. }
\end{array}
$$

The sum $\sum_{y} p^{0}(x, y)$ is $<1$ for points near the boundary, corresponding to the fact that the random walk has a nonzero probability of being killed.

Let $x \in \mathbf{P}_{++}$and consider the irreducible representation $\xi_{x}$ of $S U(n)$, with highest weight $x-\rho$. We can use Weyl's character formula (7.2) for decomposing the representation $\xi_{x} \otimes \xi_{n}$ (where $\xi_{n}$ is the defining representation of $S U(n))$.

We remark that $\frac{1}{n} \sum_{j=1}^{n} e\left(e_{j}\right)=\frac{1}{n !} \sum_{\sigma \in S_{n}} e\left(\sigma\left(e_{1}\right)\right)$. One has

$$
\begin{aligned}
\chi_{n} \chi_{x-\rho} & =\frac{1}{n}\left(e\left(e_{1}\right)+\ldots+e\left(e_{n}\right)\right) \frac{\sum_{\sigma \in S_{n}} \epsilon(\sigma) e(\sigma(x))}{\sum_{\sigma \in S_{n}} \epsilon(\sigma) e(\sigma(\rho))} \\
& =\frac{\sum_{\tau \in S_{n}} \sum_{\sigma \in S_{n}} \epsilon(\sigma) e\left(\sigma(x)+\tau\left(e_{1}\right)\right)(u)}{n ! \sum_{\sigma \in S_{n}} \epsilon(\sigma) e(\sigma(\rho))} \\
& =\frac{1}{n} \frac{\sum_{j=1}^{n} \sum_{\sigma \in S_{n}} \epsilon(\sigma) e\left(\sigma\left(x+e_{j}\right)\right)}{\sum_{\sigma \in S_{n}} \epsilon(\sigma) e(\sigma(\rho))} \\
& =\frac{1}{n} \sum_{y \in\left\{x+e_{1}, x+e_{2}, \ldots, x+e_{n}\right\} \cap \mathbf{P}_{++}} \chi_{y-\rho}
\end{aligned}
$$

since if $y=x+e_{j}$ belongs to $\mathbf{P}_{+} \backslash \mathbf{P}_{++}$then it is fixed by some reflexion in $S_{n}$ and thus the sum $\sum_{\sigma \in S_{n}} \epsilon(\sigma) e\left(\sigma\left(x+e_{j}\right)\right)$ vanishes.

For $x \in \mathbf{P}_{++}$, let us denote $h(x)$ the dimension of the representation with highest weight $x-\rho$. We conclude from the preceding computation and Proposition 6.4, that the transition probabilities for the Markov chain on $\mathbf{P}_{++}$are

$$
q(x, y)=\frac{h(y)}{h(x)} p^{0}(x, y) \quad x, y, \in \mathbf{P}_{++}
$$

Since the transition operator is unit preserving, it follows in particular that the function $h$ is a positive harmonic function with respect to the transition kernel $p^{0}$, i.e. satisfies 


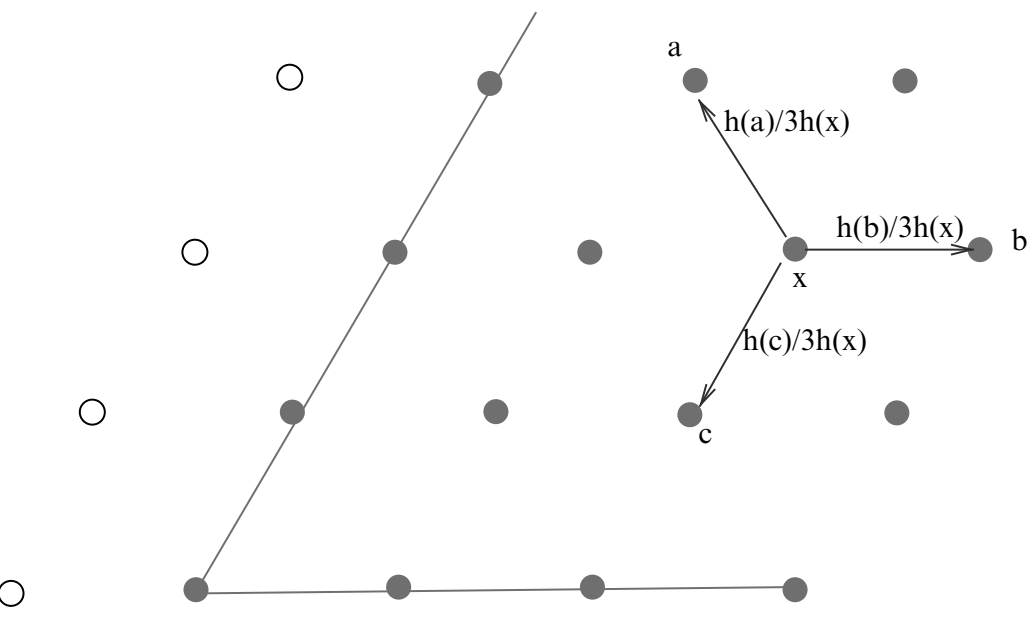

Fig. 4

$$
h(x)=\sum_{y} h(y) p^{0}(x, y) \quad \text { for all } x \in \mathbf{P}_{++},
$$

Again we draw the picture in the case of $S U(3)$.

Let us recall that, given transition (sub)probabilities $p(x, y)$ for a Markov chain, and a positive harmonic function $h$ for $p$, i.e. a function such that

$$
h(x)=\sum_{y} h(y) p(x, y) \quad \text { for all } x
$$

one calls the Markov chain with transition probabilities $\frac{h(y)}{h(x)} p(x, y)$ the Doob conditioning, or $h$-transform, of the Markov chain with transition probabilities $p$.

We can summarize the preceding discussion in the following proposition.

Proposition 7.1. The Markov chain obtained by restriction to the center is related to the random walk on the dual of the maximal torus by a killing at the exit of the Weyl chamber followed by a Doob conditioning using the dimension function on the set of highest weights.

For more information on Doob's conditioning, I refer to the books by Kemeny, Knapp and Snell [KKS], or Revuz [R].

One could ask whether this relation, between the Markov chains on the dual of the torus and on the center, holds for more general groups and positive definite functions. It turns out that the fundamental concept in this direction is that of a minuscule weight, see [B3] for more details. 


\section{Choquet-Deny Theorem for Duals of Compact Groups}

\subsection{The Choquet-Deny Theorem in an Abelian Group}

As we have seen in the preceding section, the fact that the dimension function is a positive harmonic function on the Weyl chamber plays an important role in understanding the quantum random walk on the dual of $S U(n)$. A natural question arises, is this positive harmonic function unique? We shall answer this question, which is purely a question of "classical" potential theory using the theory of quantum random walks. Actually we shall do this by extending a well known result of Choquet and Deny [CD] on harmonic measures for locally compact abelian goups. The Choquet-Deny theorem describes completely the solutions of the convolution equation

$$
\mu * \phi=\phi
$$

on a locally compact abelian group $G$, where $\mu$ is a positive finite measure, and $\phi$ is an unknown positive measure. One assumes that the subgroup generated by the support of $\mu$ is the whole group. In order to state the Choquet-Deny theorem, one needs to define the exponentials on the group $G$. These are the continuous functions $f$ on $G$, with values in $] 0,+\infty[$, which are multiplicative, i.e. satisfy

$$
f(g h)=f(g) f(h)
$$

for all $g, h \in G$. An exponential $e: G \rightarrow] 0,+\infty[$ is called $\mu$-harmonic if one has $\int_{G} e(-x) \mu(d x)=1$. The set of $\mu$-harmonic exponentials is a Borel subset of the set of all continuous functions on $G$, which we denote by $\mathcal{E}_{\mu}$. Let $\phi$ be a positive measure on $G$ of the form $\phi(d x)=e(x) d x$ where $d x$ is the Haar measure on $G$ and $e$ is a $\mu$-harmonic exponential, then one has for all positive measurable functions $f$

$$
\begin{aligned}
\int_{G} f(x) \phi * \mu(d x) & =\int_{G} \int_{G} f(x+y) \phi(d x) \mu(d y) \\
& =\int_{G} \int_{G} f(x+y) e(x) d x \mu(d y) \\
& =\int_{G} \int_{G} f(x) e(x-y) d x \mu(d y) \\
& =\int_{G} f(x) e(x) d x \int_{G} e(-y) \mu(d y) \\
& =\int_{G} f(x) \phi(d x)
\end{aligned}
$$

therefore the measure $\phi$ is $\mu$-harmonic. i.e. satisfies the equation (8.1). The Choquet-Deny theorem states that every solution is a convex combination of solutions of this kind.

Theorem 8.1 (Choquet-Deny). Assume that the subgroup generated by the support of the measure $\mu$ is $G$, then every positive measure $\phi$, solution of the convolution equation (8.1), is absolutely continuous with respect ot the Haar measure on $G$, and its density has a unique representation as an integral 


$$
\frac{d \phi(x)}{d x}=\int_{\mathcal{E}_{\mu}} e(x) d \nu_{\phi}(e)
$$

where $\nu_{\phi}$ is some finite positive measure on $\mathcal{E}_{\mu}$.

This result contains in particular the determination of all positive harmonic functions for the transition operator associated with the measure $\mu$, indeed if $h$ is such a positive $\mu$-harmonic function, then the measure $h(-x) d x$ is a $\mu$-harmonic measure.

Note that a locally compact abelian group corresponds to a commutative and cocommutative Hopf $C^{*}$-algebra. What we shall do next is to extend this theorem to (a class of) cocommutative Hopf $C^{*}$-algebras. This will allow us to answer the question about the uniqueness of the positive harmonic function. Before we state our analogue of the Choquet-Deny theorem on the dual of a compact group, we will first clarify some points about states and weights.

\subsection{Some Further Properties of Duals of Compact Groups}

Let $G$ be a compact group and $\mathcal{A}(G)$ be its von Neumann algebra. This von Neumann algebra has a structure of cocommutative Hopf-von Neumann algebra for the coproduct $\Delta$, counit $\varepsilon$ and antipode $i$ given respectively by continuous linear extension of

$$
\Delta\left(\lambda_{g}\right)=\lambda_{g} \otimes \lambda_{g} \quad \varepsilon\left(\lambda_{g}\right)=\delta_{e, g} \quad i\left(\lambda_{g}\right)=\lambda_{g^{-1}} .
$$

We have seen in section 4.3 that the convolution of two finite normal weights $\mu$ and $\nu$ on $\mathcal{A}(G)$ is defined by the formula $\mu * \nu=(\mu \otimes \nu) \circ \Delta$. Assume now that $\mu$ is a positive finite weight and $\phi$ is positive, then we can write $\phi$ as a sum of finite weights $\phi=\sum_{\chi} \phi_{\chi}$ with respect to the restrictions of $\phi$ to the subalgebras $M_{\chi}$. The sum $\sum_{\chi} \nu * \phi_{\chi}$ is then a sum of positive finite weights and thus it defines a (not necessarily normal) weight on $\mathcal{A}(G)$. We can therefore consider the equation (8.1) where this time $\mu$ and $\phi$ are positive normal weights, with $\mu$ finite.

Next we define the notion of exponential. The following definition is a straighforward extension of the definition in the case of abelian groups.

Definition 8.2. A group-like element is a non zero element $f$ of $\hat{\mathcal{A}}(G)$, such that

$$
\Delta(f)=f \otimes f .
$$

If this element is positive, then we call it an exponential.

If $\mu$ is a finite weight, $f$ is an exponential and $\mu(i(f))=1$ then we call $f$ a $\mu$-harmonic exponential.

Let $f, g, h$ be positive elements in $\hat{\mathcal{A}}(G)$ then one has the identity

$$
\operatorname{tr}(f \otimes g \Delta(h))=\operatorname{tr}(i(h) \otimes g \Delta(i(f)))
$$


which is easily checked on coefficient functions. Let $g \in \hat{\mathcal{A}}(G)$ be associated to $\mu$, i.e. $\mu()=.\operatorname{tr}(g$.$) , then it follows from 8.2$ that for all $h$

$$
\begin{aligned}
\phi * \mu(h) & =\operatorname{tr}(f \otimes g \Delta(h)) \\
& =\operatorname{tr}(i(h) \otimes g \Delta(i(f))) \\
& =\operatorname{tr}(i(h) i(f)) \operatorname{tr}(g i(f)) \\
& =\phi(h)
\end{aligned}
$$

Thus if $f$ is a $\mu$-harmonic exponential, and $\phi$ is the weight associated with $f$, then $\phi$ satisfies the equation (8.1).

\subsection{The Analogue of the Choquet-Deny Theorem}

We shall assume that the state $\nu$ satisfies a non degeneracy condition which is the analogue, in the noncommutative setting, of the requirement that the support of the measure generates the whole group. This condition states that for every finite weight $\rho$ on $\mathcal{A}(G)$ which is supported by some algebra $M_{\chi}$ for $\chi \in \hat{G}$, there exists an integer $n \geq 1$ and a constant $c>0$ such that $c \nu^{* n} \geq \rho$.

Theorem 8.3. Let $\phi$ be a $\nu$-harmonic weight, then there exists a unique finite positive measure on the set of $\nu$-harmonic exponentials such that

$$
\phi=\int_{\mathcal{E}_{\mu}} e d m_{\phi}(e)
$$

Sketch of proof. We let $\mathcal{S}_{\nu}$ be the convex cone of normal weights satisfying

$$
\phi * \nu \leq \phi
$$

This cone is a closed subset of $\hat{\mathcal{A}}$, and we can use Choquet's integral representation theorem to conclude that any element of this cone can be written as the barycenter of a measure supported on the set of its extremal rays. Now one can analyze the extremal rays of this set, and see that such an extremal ray consists in multiples of an exponential. The uniqueness argument comes from the existence of an algebra of functions on the cone, which separates points, see [B4] for details.

\subsection{Examples}

For a finite group, the set of exponentials is reduced to the identity.

If the state $\nu$ is tracial, then there exists only one $\nu$-harmonic exponential, namely the identity. In the case of the group $S U(n)$, the exponentials are in one to one correspondence with the positive elements in $S L(n, \mathbb{C})$. 
From these results we deduce that the positive harmonic function $h$ of Proposition 7.1 was unique. Indeed in order to prove that a function $h$ is the unique (up to a multiplicative constant) positive harmonic function for a transition kernel $p$, it is enough to check that all the positive harmonic functions for the relativized kernel $\frac{h(y)}{h(x)} p(x, y)$ are constant, which is what the Choquet-Deny theorem tells us.

\section{The Martin Compactification of the Dual of $S U(2)$}

In the preceding section we have seen that the classical Choquet-Deny theorem about solutions of convolution equations has an analogue in duals of compact groups. This allows one to give an explicit description of all $\mu$-harmonic positive functions for a finite positive measure $\mu$ on a commutative group. By the Choquet-Deny theorem the positive $\mu$-harmonic functions admit a unique integral representation in terms of minimal $\mu$-harmonic functions, and these minimal harmonic functions can be identified with exponentials. The next natural question in this line of ideas is to describe the Martin compactification associated to a random walk with values in $\mathbb{Z}^{n}$. This Martin compactification provides a way to attach a boundary to the space $\mathbb{Z}^{n}$ in order to obtain a compact space, where the boundary is naturally identified with the set of minimal $\mu$-harmonic functions. The Martin compactification of $\mathbb{Z}^{n}$ was computed by Ney and Spitzer in a classical paper [NS], where they showed that it consists in adding a sphere at infinity, and identifying this sphere with the set of minimal harmonic functions with the help of the Gauss map. In this section we will describe the Martin compactification of some quantum random walks with values in the dual of $S U(2)$. We will first recall some basic facts about classical Martin boundaries, then describe Ney and Spitzer's theorem, before going to the case of the dual of $S U(2)$.

\subsection{The Martin Compactification for Markov Chains}

Consider a Markov chain on a countable state space $E$. The Markov chain has transition subprobabilities $p(x, y), x, y \in E$, i.e. we have $\sum_{y} p(x, y) \leq 1$, so that the kernel is submarkovian and the process may die in a finite time. There is an associated transition operator given by

$$
P f(x)=\sum_{y \in E} p(x, y) f(y)
$$

and the iterated operator is given by $n$-step transition probabilities

$$
P^{n} f(x)=\sum_{E} p_{n}(x, y) f(y)
$$


We assume that the associated Markov chain is transient, so that the potential

$$
U=\sum_{n=0}^{\infty} P^{n}
$$

is finite, i.e. the function

$$
u(x, y)=\sum_{n=0}^{\infty} p_{n}(x, y)<\infty
$$

Let us choose an initial distribution $r(d x)$ such that the function $r U(y)=$ $\sum_{E} u(x, y) r(d x)$ is everywhere $>0$. The Martin kernel is defined by

$$
k(x, y)=\frac{u(x, y)}{r U(y)}
$$

It follows from the Harnack inequalities that the functions $k(x,$.$) form a uni-$ formly continuous family on $E$. The Martin compactification of the Markov chain is the smallest compact topological space $\bar{E}_{u}$, which contains $E$ as a dense subset, and such that these functions extend continuously to the boundary $\partial E_{u}=\bar{E}_{u} \backslash E$. This space exists because the functions $k(x,$.$) separate$ the points of $E$ and because of the uniform continuity. For any $\xi \in \partial E_{u}$ the function $x \mapsto k(x, \xi)$ is a $p$-harmonic function. Recall that a positive $p$ harmonic function $f$ on $E$ is called minimal if for every positive $p$-harmonic function $g$ satisfying $g \leq C f$ for some $C>0$, one has actually $g=c f$ for some constant $c$. One can prove that any minimal $p$-harmonic function $f$, which is $r$-integrable, is a multiple of $k(., \xi)$ for some $\xi \in \partial E_{u}$. The subset $\partial E_{m}$ of $\xi \in \partial E_{u}$ such that $k(., \xi)$ is minimal is a Borel subset, and one can prove that any positive $p$-harmonic function $f$, which is $r$-integrable, admits a representation

$$
f=\int_{\partial E_{u}} k(., \xi) d m_{f}(\xi)
$$

with a unique positive measure $m_{f}$.

\subsection{The Martin Compactification of $\mathbb{Z}^{d}$}

When the Markov chain is a random walk on $\mathbb{Z}^{d}$, with increments distributed as $\mu$, a (sub)probability measure on $\mathbb{Z}^{d}$, we have seen that every positive $\mu$ harmonic function admits an integral representation in terms of exponentials. When the increments of the random walk are integrable, Ney and Spitzer have determined explicitly the Martin compactification of $\mathbb{Z}^{d}$, which we shall now describe. Let $\phi: \mathbb{R}^{d} \rightarrow[0,+\infty]$ be the function

$$
\phi(x)=\sum_{y \in \mathbb{Z}^{d}} e^{\langle x, y\rangle} \mu(d y)
$$


We assume that it is finite in a neighbourhood of the set

$$
\mathcal{E}_{\mu}=\{x \mid \phi(x)=1\}
$$

Then the set $\mathcal{E}_{\mu}$ is the boundary of the convex set $\{x \mid \phi(x) \leq 1\}$ and it is either reduced to a point or homeomorphic to a sphere. In the latter case the homeomorphism can be expressed thanks to the Gauss map as

$$
\frac{\nabla \phi}{\|\nabla \phi\|}
$$

Since $\phi$ is convex this is a homeomorphism from $\mathcal{E}_{\mu}$ onto the unit sphere. Ney and Spitzer proved that the Martin compactification is homeomorphic to the usual compactification of $\mathbb{Z}^{d}$ by a sphere at infinity, where the identification between the sphere and the set of minimal $\mu$-harmonic functions is provided by the map above.

\subsection{Noncommutative Compactifications}

Before we investigate the problem of finding an analogue of the Ney-Spitzer theorem for the dual of $S U(2)$ let us translate in the noncommutative language the notion of a compactification of a topological space. So let $X$ be a topological space, and $\bar{X}$ a compact space such that $X \subset \bar{X}$ is a dense open subset, and $\partial \bar{X}=\bar{X} \backslash X$ is the boundary, then $C(\bar{X})$ is can be identified with a subalgebra of $C_{b}(X)$, the algebra of all bounded continuous functions on $X$, and one has an exact sequence

$$
0 \rightarrow C_{0}(X) \rightarrow C(\bar{X}) \rightarrow C(\partial X) \rightarrow 0
$$

where the first map is the continuous extension, to $\bar{X}$, by 0 on the boundary, of a function on $X$, and the second map is the restriction to a closed subset. A compactification of $C_{0}(X)$ is thus given by a certain commutative $C^{*}$ subalgebra of the multiplier algebra of $C_{0}(X)$, containing $C_{0}(X)$. In the case of the Martin compactification this subalgebra is just the algebra generated by $C_{0}(X)$ and by the functions $y \mapsto k(x, y)$. The vector space generated by functions of the form $y \mapsto k(x, y)$ can be identified with the image of the Martin kernel, considered as an integral operator $f \mapsto \sum_{x \in E} f(x) k(x, y)$. It is this interpretation of the Martin kernel that has a natural noncommutative analogue.

\subsection{The Martin Kernel for the Quantum Random Walk}

We consider a central quantum random walk, therefore we have a positive definite central function $\phi$ on $S U(2)$ such that $\phi(e) \leq 1$, and the associated 
state $\nu$ on $\mathcal{A}(S U(2))$. We have seen that it has an associated transition kernel given by the completely positive map $\Phi_{\nu}=(I \otimes \nu) \circ \Delta$. This map acts as $\lambda_{g} \mapsto \phi(g) \lambda_{g}$ on the generators $\lambda_{g}$ of the von Neumann algebra $\mathcal{A}(S U(2))$. We know that if $\phi(e)=1$ then there exists only one $\phi$-harmonic weight, namely the one given by the function 1 . In this case we expect that the Martin boundary will be given by a one point compactification, which consists just in adding a unit to the algebra, so in order to avoid this case, we shall assume here that $\phi(e)<1$.

We can consider the associated potential which is equal to $U=\sum_{n=0}^{\infty} \Phi_{\nu}^{n}$, and which acts by multiplication by the function $\sum_{n=0}^{\infty} \phi^{n}=\frac{1}{1-\phi}$. We shall consider the action of the potential on the space of coefficients i.e. the direct sum $\oplus_{\chi} M_{\chi}$ where each element of this space can be identified with a polynomial function on $S U(2)$ acting by convolution, i.e. by the operator $\int_{S U(2)} p(g) \lambda_{g} d g$. Then the Martin kernel will be defined, by analogy with the case of classical Markov chains, by

$$
p \mapsto \frac{\int_{S U(2)} \frac{p(g)}{1-\phi(g)} \lambda_{g} d g}{\int_{S U(2)} \frac{1}{1-\phi(g)} \lambda_{g} d g}
$$

Note that the operator $\int_{S U(2)} \frac{1}{1-\phi(g)} \lambda_{g} d g$ lies in the center of $\mathcal{A}(S U(2))$, therefore there is no ambiguity in the quotient of the preceding formula.

\subsection{Pseudodifferential Operators of Order Zero and the Martin Compactification}

In order to find the Martin compactification of the quantum random walk, we shall identify the $C^{*}$-algebra generated by $C^{*}(S U(2))$ and by the image of the Martin kernel, and show that it gives rise to a three terms exact sequence. We will first exhibit a certain exact sequence

$$
0 \rightarrow C^{*}(S U(2)) \rightarrow \mathcal{M} \rightarrow C\left(S^{2}\right) \rightarrow 0
$$

where $\mathcal{M}$ is our sought for Martin compactification, and $C\left(S^{2}\right)$ is the algebra of continuous functions on the two dimensional sphere $S^{2}$. For this we consider the three operators in $\hat{\mathcal{A}}(S U(2))$ associated with Pauli matrices, which we call $X, Y, Z$, and the Casimir operator $C=X^{2}+Y^{2}+Z^{2}+I$ which acts by $(n+1)^{2}$ on the space of coefficients of the $n$-dimensional representation of $S U(2)$, and build three operators

$$
x=X C^{-1 / 2}, y=Y C^{-1 / 2}, z=Z C^{-1 / 2}
$$

Clearly these operators are self-adjoint and bounded. Using the commutation relations (3.1) one can see that $[x, y],[x, z],[y, z]$ and $x^{2}+y^{2}+z^{2}-I$ are 
compact operators on $L^{2}(S U(2))$. It follows that there exists a map from the algebra generated by $x, y, z$ to the algebra of polynomial function on the sphere, sending $x, y, z$ to the three coordinate functions, and this map vanishes on the compact operators. Actually this map extends by continuity to the $C^{*}$ algebra generated by $x, y$ and $z$ and yields the exact sequence (9.1). One can interpret also the algebra $\mathcal{M}$ as the algebra of right invariant pseudo differential operators of order zero on $S U(2)$, then the map $\mathcal{M} \rightarrow C\left(S^{2}\right)$ of (9.1) is the principal symbol map (see [B5]). Once we have introduced the exact sequence above, we can state the theorem which is the analogue, for central states on the dual of $S U(2)$, of the Ney-Spitzer theorem.

Theorem 9.1. The $C^{*}$ algebra generated by the image of the Martin kernel is the algebra $\mathcal{M}$. The Martin kernel yields a section $K: C\left(S^{2}\right) \rightarrow \mathcal{M}$.

The proof of the theorem relies on a detailed analysis of the Clebsch-Gordan formulas, see [B5].

Recently the problem of the Martin or Poisson boundary have been considered for quantum groups, see [Col], [I], [INT]

\section{Central Limit Theorems for the Bernoulli Random Walk}

Just as in the classical case there exists central limit theorems for the Bernoulli random walk, however the noncommutativity here plays an important role, and according to whether the state we chose is central or not the limit is quite different.

\subsection{The Case of a Central State}

We consider the triple of processes $\left(X_{n}, Y_{n}, Z_{n}\right)_{n \geq 1}$ constructed in section (3.1). We use the tracial state to contruct the product, $M_{2}(\mathbb{C})^{\otimes \infty}$ which is thus endowed with the tracial state $\sigma=\left(\frac{1}{2} T r\right)^{\otimes \infty}$. We renormalize the three processes according to

$$
X_{t}^{(\lambda)}=\frac{X_{[\lambda t]}}{\sqrt{\lambda}}, \quad Y_{t}^{(\lambda)}=\frac{Y_{[\lambda t]}}{\sqrt{\lambda}}, \quad Z_{t}^{(\lambda)}=\frac{Z_{[\lambda t]}}{\sqrt{\lambda}}
$$

where $[x]$ is the integer part of $x$. This triple of processes converges when $\lambda \rightarrow \infty$, in the sense of moments, towards a three dimensional Bownian motion.

Theorem 10.1. Let $\left(X_{t}, Y_{t}, Z_{t}\right)_{t \geq 0}$ be a three dimensional Brownian motion, then for any polynomial in $3 n$ noncommuting indeterminates $P$, and all times $t_{1}, \ldots, t_{n}$, one has 


$$
\begin{gathered}
\lim _{\lambda \rightarrow \infty} \sigma\left(P\left(X_{t_{1}}^{(\lambda)}, Y_{t_{1}}^{(\lambda)}, Z_{t_{1}}^{(\lambda)}, \ldots, X_{t_{n}}^{(\lambda)}, Y_{t_{n}}^{(\lambda)}, Z_{t_{n}}^{(\lambda)}\right)\right)= \\
E\left[P\left(X_{t_{1}}, Y_{t_{1}}, Z_{t_{1}}, \ldots, X_{t_{n}}, Y_{t_{n}}, Z_{t_{n}}\right)\right]
\end{gathered}
$$

Let us sketch the proof of this result. It is enough to prove the result for monomials. First we see that for any real coefficients $x, y, z$ the process $x X_{t}^{(\lambda)}+y Y_{t}^{(\lambda)}+z Z_{t}^{(\lambda)}$ converges towards real Brownian motion. By polarization this implies that for any monomial in $X, Y, Z$ the sum over all monomials with the same total partial degrees in $X, Y, Z$ converges towards the required limit. Consider two monomials of the form $M_{1} X_{t}^{(\lambda)} Y_{s}^{(\lambda)} M_{2}$ and $M_{1} Y_{s}^{(\lambda)} X_{t}^{(\lambda)} M_{2}$, their difference is, thanks to the commutation relations, $M_{1} Z_{s \wedge t}^{(\lambda)} M_{2} / \sqrt{\lambda}$. This is a monomial of smaller degree, with a factor $1 / \sqrt{\lambda}$. We conclude, by induction on degrees of monomials, that the difference betwen the expectations of the two monomials $M_{1} X_{t}^{(\lambda)} Y_{s}^{(\lambda)} M_{2}$ and $M_{1} Y_{s}^{(\lambda)} X_{t}^{(\lambda)} M_{2}$ converges to 0 as $\lambda \rightarrow \infty$. It follows that the expectations of all monomials with the same partial degrees in variables converge to the same limit.

We observe that the spin process, normalized by $S_{[\lambda t]} / \sqrt{\lambda}$ converges in distribution to a three dimensional Bessel process as $\lambda \rightarrow \infty$.

\subsection{The Case of a Pure State}

Now we consider the quantum Bernoulli random walk with the pure state given by the vector $e_{0}$. We shall consider the convergence of the moments of the triple of processes

$$
\left(X_{t}^{(\lambda)}, Y_{t}^{(\lambda)}, Z_{t}^{(\lambda)}\right)=\left(\frac{X_{[\lambda t]}}{\sqrt{\lambda}}, \frac{Y_{[\lambda t]}}{\sqrt{\lambda}}, \frac{Z_{[\lambda t]}}{\lambda}\right) ; t \geq 0 .
$$

We shall prove that there exists operators $\left(X_{t}, Y_{t}, Z_{t}\right)_{t \geq 0}$ on some Hilbert space $H$ with a vector $\Omega \in H$, such that for every polynomial in noncommuting indeterminates $P\left(X_{t_{1}}, Y_{t_{1}}, Z_{t_{1}}, \ldots, X_{t_{n}}, Y_{t_{n}}, Z_{t_{n}}\right)$ one has

$$
\begin{gathered}
\left.\lim _{n \rightarrow \infty}\left\langle P\left(X_{t_{1}}^{(\lambda)}, Y_{t_{1}}^{(\lambda)}, Z_{t_{1}}^{(\lambda)}, \ldots, X_{t_{n}}^{(\lambda)}, Y_{t_{n}}^{(\lambda)}, Z_{t_{n}}^{(\lambda)}\right) e_{0}^{\infty}, e_{0}^{\infty}\right\rangle\right)= \\
\left\langle P\left(X_{t_{1}}, Y_{t_{1}}, Z_{t_{1}}, \ldots, X_{t_{n}}, Y_{t_{n}}, Z_{t_{n}}\right) \Omega, \Omega\right\rangle
\end{gathered}
$$

For this we shall first investigate the case where $n=1, t_{1}=1$, thus we have just three operators $\left(\frac{X_{n}}{\sqrt{n}}, \frac{Y_{n}}{\sqrt{n}}, \frac{Z_{n}}{n}\right)$ and let $n \rightarrow \infty$. Let $H$ be a Hilbert space with a countable orthonormal basis $\varepsilon_{0}, \ldots, \varepsilon_{n}, \ldots$, and let us define operators, with domain the algebraic sum $\oplus_{i=0}^{n} \mathbb{C} \varepsilon_{i}$, by the formula

$$
\begin{aligned}
& a^{+}\left(\varepsilon_{i}\right)=\sqrt{j+1} \varepsilon_{j+1} \\
& a^{-}\left(\varepsilon_{j}\right)=\sqrt{j} \varepsilon_{i-1}
\end{aligned}
$$


Theorem 10.2. For any polynomial in three noncommutative indeterminates one has

$$
\lim _{n \rightarrow \infty}\left\langle P\left(\frac{X_{n}}{\sqrt{n}}, \frac{Y_{n}}{\sqrt{n}}, \frac{Z_{n}}{n}\right) e_{0}^{\otimes n}, e_{0}^{\otimes n}\right\rangle=\left\langle P\left(a^{+}+a^{-}, \frac{1}{i}\left(a^{+}-a^{-}\right), I\right) \varepsilon_{0}, \varepsilon_{0}\right\rangle
$$

The proof is immediate by inspection of the formula (3.2) and comparison with (10.1).

Observe that one has the adjointness relations

$$
\left\langle a^{+} u, v\right\rangle=\left\langle u, a^{-} v\right\rangle
$$

for all $u, v$ in the domain. It follows that the operators $a^{+}+a^{-}$and $\frac{1}{i}\left(a^{+}-a^{-}\right)$ are unbounded symmetric operators on $H$, and thus are closable. We will see below that they have self-adjoint extensions. They satisfy the commutation relation

$$
\left[a^{+}, a^{-}\right]=-I
$$

on their common domain, spanned by the vectors $\varepsilon_{i}$.

The operators $a^{+}, a^{-}$thus obtained are well known under the name of creation and annihilation operators for the quantum harmonic oscillator. One can give a natural model for these operators using a gaussian random variable. For this, remark that the distribution of the operator $a^{+}+a^{-}$is gaussian. This follows easily from the fact that each $X_{n}$ follows a standard binomial distribution, and the convergence of this binomial distribution to the gaussian distribution, by the de Moivre-Laplace theorem. This property of the operator $a^{+}+a^{-}$, and the fact that $\varepsilon_{0}$ is a cyclic vector for $a^{+}+a^{-}$, i.e. the vectors $\left(a^{+}+a^{-}\right)^{n} \varepsilon_{0}$ span a dense subspace of $H$, allows us to identify the space $H$ in a natural way with the $L^{2}$ space of a gaussian random variable. The vectors $\varepsilon_{n}$ can be obtained from the vectors $\left(a^{+}+a^{-}\right)^{n}$ by the Gram-Schmidt orthogonalization procedure. It is well known that, for a gaussian variable $X$, the polynomials obtained by the Gram-Schmidt orthonormalization process from the sequence $X^{n}$ are the Hermite polynomials. Thus when we identify the space $H$ with the $L^{2}$ space of the gaussian measure on $\mathbb{R}$ the vectors $\varepsilon_{n}$ become identified with the Hermite polynomials. Then the operator $a^{-}$is identified with $\frac{d}{d x}$ and the operator $a^{+}$with $x-\frac{d}{d x}$.

The product $a^{+} a^{-}$has eigenvalues $0,1,2, \ldots$ corresponding to the the respective eigenvectors $\varepsilon_{0}, \varepsilon_{1}, \ldots$ It is known as the number operator in quantum field theory.

We will in the next section systematize the construction above and show how to deduce the limit of the renormalized quantum random walk to a quantum Brownian motion. 


\subsection{Fock Spaces}

Let $H$ be a complex Hilbert space. For each integer $n$ the symmetric group $S_{n}$ acts on $H^{\otimes n}$ by permutation of the factors in the tensor product.

Definition 10.3. We denote $H^{\circ n}$ the subspace of $H^{\otimes n}$ formed by vectors invariant under the action of $S_{n}$.

If $h_{1}, \ldots, h_{n} \in H$ then we let

$$
h_{1} \circ \ldots \circ h_{n}=\frac{1}{\sqrt{n !}} \sum_{\sigma \in S_{n}} h_{\sigma(1)} \otimes \ldots \otimes h_{\sigma(n)}
$$

which is a multiple of the orthogonal projection of $h_{1} \otimes \ldots \otimes h_{n}$ on $H^{\circ n}$. One has

$$
\left\langle h_{1} \ldots \circ h_{n}, h_{1}^{\prime} \circ \ldots \circ h_{n}^{\prime}\right\rangle=\sum_{\sigma \in S_{n}} \prod_{i=1}^{n}\left\langle h_{i}, h_{\sigma(i)}^{\prime}\right\rangle
$$

The Fock space built on $H$ is the Hilbert space direct sum

$$
\Gamma(H)=\oplus_{n=0}^{\infty} H^{\circ n}
$$

where $H^{\circ 0}$ is a one dimensional Hilbert space spanned by a unit vector $\Omega$, called the vacuum vector of the Fock space. The algebraic direct sum $\oplus_{\text {alg }, n=0}^{\infty} H^{\circ n}$, denoted by $\Gamma_{0}(H)$, is a dense subspace of $\Gamma(H)$.

For every $h \in H$ we define the exponential vector associated with $h$ by

$$
\xi(h)=\sum_{n=0}^{\infty} \frac{h^{\circ n}}{n !}
$$

one has

$$
\left\langle\xi(h), \xi\left(h^{\prime}\right)\right\rangle=e^{\langle h, k\rangle}
$$

furthermore the vectors $\xi(h) ; h \in H$ form a linearly free subset, generating algebraically a dense subspace of $\Gamma(H)$.

If the space $H$ is written as the orthogonal direct sum of two Hilbert subspaces $H=H_{1} \oplus H_{2}$, then there is a canonical isomorphism

$$
\Gamma(H) \sim \Gamma\left(H_{1}\right) \otimes \Gamma\left(H_{2}\right)
$$

which can be obtained, for example, by identifying the exponential vector $\xi\left(v_{1}+v_{2}\right) \in \Gamma(H)$, where $v_{1} \in H_{1}$ and $v_{2} \in H_{2}$, with the vector $\xi\left(v_{1}\right) \otimes \xi\left(v_{2}\right) \in$ $\Gamma\left(H_{1}\right) \otimes \Gamma\left(H_{2}\right)$.

Let $h \in H$, we define two operators on the domain $\Gamma_{0}(H)$ by

$$
\begin{aligned}
& a_{h}^{+}\left(h_{1} \circ \ldots \circ h_{n}\right)=h \circ h_{1} \circ \ldots \circ h_{n} \\
& a_{h}^{-}\left(h_{1} \circ \ldots \circ h_{n}\right)=\sum_{i=1}^{n}\left\langle h_{i}, h\right\rangle h_{1} \circ \ldots \circ \hat{h}_{i} \circ \ldots \circ h_{n}
\end{aligned}
$$


Operators of the form $a_{h}^{+}$are called creation operators while the $a_{h}^{-}$are called annihilation operators. When $H$ is one dimensional, generated by a unit vector $u$ there is a natural identification of $\Gamma(H)$ with the Hilbert space of the preceding section where the vector $u^{\circ n}$ is identified with $\sqrt{n !} \epsilon_{n}$. Then the operators $a_{u}^{+}$and $a_{u}^{-}$coincide with $a^{+}$and $a^{-}$.

Another operator of interest on $\Gamma(H)$ is the number operator $\Lambda$ which has eigenvalue $n$ on the subspace $H^{\circ n}$. If $\left(e_{i}\right)_{i \in I}$ is an orthonormal basis in $H$, then the number operator $\Lambda$ has the expansion

$$
\Lambda=\sum_{i} a_{e_{1}}^{+} a_{e_{i}}^{-} .
$$

One can see that the creation and annihilation operators satisfy the adjoint relation

$$
\left\langle a_{h}^{+} u, v\right\rangle=\left\langle u, a_{h}^{-} v\right\rangle \quad h, u, v \in \Gamma_{0}(H)
$$

as well as the commutation relation

$$
\left[a_{h}^{+}, a_{k}^{-}\right]=-\langle h, k\rangle I
$$

on the domain $\Gamma_{0}(H)$. In particular they are closable, and it is easy to see that the exponential vectors belong to the domain of their closure, with

$$
a_{h}^{+} \xi\left(h^{\prime}\right)=\frac{d}{d t} \xi\left(h^{\prime}+t h\right)_{t=0} \quad a_{h}^{-} \xi\left(h^{\prime}\right)=\left\langle h^{\prime}, h\right\rangle \xi(h)
$$

We shall see that the real part of the creation operator $P_{h}=a_{h}^{+}+a_{h}^{-}$has a selfadjoint extension, as well as its imaginary part $Q_{h}$. For this we consider the following Weyl operators, given on the vector space generated by exponential vectors by the formula

$$
W_{u} \xi(h)=\xi(h+u) e^{-\langle h, u\rangle-\frac{1}{2}\langle u, u\rangle}
$$

It is easy to check that

$$
\left\langle W_{u} \xi(h), W_{u} \xi\left(h^{\prime}\right)\right\rangle=\left\langle\xi(h), \xi\left(h^{\prime}\right)\right\rangle
$$

for all $u, v, h, h^{\prime} \in H$, therefore the operators $W_{u}$ extend to unitary operators on $\Gamma(H)$, furthermore

$$
W_{u} W_{v}=W_{u+v} e^{-i \Im\langle u, v\rangle}
$$

and for any $u \in H$ the operators $\left(W_{i t u} ; t \in \mathbb{R}\right)$ form a one parameter group of unitary operators, whose generator is given by $P_{u}$ on exponential vectors. Similarly, the operators $\left(W_{t u} ; t \in \mathbb{R}\right)$ form a one parameter group of unitary operators, whose generator is given by $Q_{u}$, and more generally for $\theta \in[0,2 \pi[$ the vectors $\left(W_{e^{i \theta} t u} ; t \in \mathbb{R}\right)$ form a one parameter group of unitary operators, whose generator is given by $\cos \theta Q_{u}+\sin \theta P_{u}$. 
It follows from Stone's theorem that $P_{u}, Q_{u}$, and all their linear combinations, have a self adjoint extension. These operators satisfy the commutation relation

$$
\left[P_{u}, Q_{v}\right]=-2 i \Re\langle u, v\rangle .
$$

We observe that if $H$ splits as an orthogonal direct sum $H=H_{1} \oplus H_{2}$ and $u \in H_{1}$, then the operator $W_{u}$ admits a decomposition $W_{u}=W_{u} \otimes I$ in the decomposition (10.2).

Let now $K \subset H$ be a real Hilbert subspace such that $\Im\langle u, v\rangle=0$ for all $u, v \in K$ (if $K$ is maximal, it is called a Lagrangian subspace), then by (10.5) the unitary operators $W_{i u} ; u \in K$ form a commutative family, and the generators $P_{u}, u \in K$ of the one parameter subgroups $\left(W_{i t u} ; t \in \mathbb{R}\right)$ form a commuting family of self-adjoint operators with common dense domain $\Gamma_{0}(H)$. We can therefore investigate the joint distribution of these operators.

Proposition 10.4. The operators $P_{u}$, for $u \in K$ form a gaussian family with covariance $\left\langle P_{u}, P_{v}\right\rangle=\langle u, v\rangle$.

The operators $Q_{u}$, for $u \in K$ form a gaussian family with covariance $\left\langle Q_{u}, Q_{v}\right\rangle=\langle u, v\rangle$.

For the proof it is enough to prove that any linear combination of these operators has a gaussian distribution with the right variance, i.e. that $P_{u}$ is a gaussian with variance $\langle u, u\rangle$. For this one computes the Fourier transform

$$
\left\langle e^{i P_{u}} \Omega, \Omega\right\rangle=\left\langle e^{i P_{u}} \xi(0), \xi(0)\right\rangle=\left\langle\xi(u) e^{-\frac{1}{2}\langle u, u\rangle}, \xi(0)\right\rangle=e^{-\frac{1}{2}\langle u, u\rangle}
$$

The proof for the operators $Q_{u}$ is similar.

We let now $H=L^{2}\left(\mathbb{R}_{+}\right)$, and take as Lagrangian subspace the subspace of real valued functions. Then the family $\left(P_{t}:=P_{1_{[0, t]}} ; t \geq 0\right)$ has the covariance $\left\langle P_{t}, P_{s}\right\rangle=s \wedge t$, and thus has the distribution of a real brownian motion. The same is true of the operators $Q_{t}:=\frac{1}{i}\left(a_{1_{[0, t]}^{+}}^{+}-a_{1_{[0, t]}}^{-}\right)$which form another Brownian motion satisfying the commutation relations

$$
\left[P_{t}, Q_{t}\right]=2 i t
$$

We shall call the pair $\left(P_{t}, Q_{t}\right)_{t \geq 0}$ of continuous time processes a noncommutative brownian motion.

We can now state the limit result we had in view in the beginning of this section.

Theorem 10.5. For any polynomial $P$ in noncommuting variables, one has

$$
\begin{gathered}
\lim _{\lambda \rightarrow \infty}\left\langle\left(P\left(X_{t_{1}}^{(\lambda)}, Y_{t_{1}}^{(\lambda)}, Z_{t_{1}}^{(\lambda)}, \ldots, X_{t_{n}}^{(\lambda)}, Y_{t_{n}}^{(\lambda)}, Z_{t_{n}}^{(\lambda)}\right) e_{0}^{\infty}, e_{0}^{\infty}\right\rangle\right)= \\
\left.\left.\left\langle P\left(P_{t_{1}}, Q_{t_{1}}, t_{1} . I, \ldots, P_{t_{n}}, Q_{t_{n}}, t_{n} . I\right)\right) \Omega, \Omega\right\rangle\right]
\end{gathered}
$$

The proof is an elaboration of the proof we gave for one time. 
We shall see in the next section that, again, one can interpret this pair of processes as a noncommutative Markov process with values in a noncommutative space.

The noncommutative Brownian motion is the basis of a theory of noncommutative stochastic integration which has been developped by Hudson and Parthasarathy, see e.g. [Pa].

\section{The Heisenberg Group and the Noncommutative Brownian Motion}

The Heisenberg group is the set $\mathcal{H}=\mathbb{C} \times \mathbb{R}$ endowed with the group law

$$
(z, w) \star\left(z^{\prime}, w^{\prime}\right)=\left(z+z^{\prime}, w+w^{\prime}+\Im\left(z \bar{z}^{\prime}\right)\right)
$$

This is a nilpotent group, its center being $\{0\} \times \mathbb{R}$, and the Lebesgue measure on $\mathbb{C} \times \mathbb{R}$ is a left and right Haar measure for this group.

The Weyl operators defined in the preceding section on a Fock space $\Gamma(\mathbb{C})$ define unitary representations of the group $\mathcal{H}$, by setting, for $\tau \in \mathbb{R}^{*}$,

$$
\rho_{\tau}(z, w)=W_{z \tau^{1 / 2}} e^{i \tau w}
$$

if $\tau>0$ and

$$
\rho_{\tau}(z, w)=W_{\bar{z}|\tau|^{1 / 2}} e^{i \tau w}
$$

if $\tau<0$.

Another family of representations is given by the one dimensional characters

$$
\rho_{\xi}(z, w)=e^{i \Re(z \bar{\xi})}
$$

for $\xi \in \mathbb{C}$.

All these representations are irreducible, are non equivalent and they exhaust the family of equivalence classes of irreducible representations of $\mathcal{H}$.

We consider the $C^{*}$ algebra of $\mathcal{H}$, which is the $C^{*}$-algebra generated by the convolution algebra $L^{1}(\mathcal{H})$ on $L^{2}(\mathcal{H})$. This algebra is a sub $C^{*}$-algebra of $B\left(L^{2}(\mathcal{H})\right)$. Let us denote $z=q+i p$ then the Lie algebra of $\mathcal{H}$ is composed of the vector fields

$$
\frac{\partial}{\partial w} ; \quad \frac{\partial}{\partial q}+p \frac{\partial}{\partial w} ; \quad \frac{\partial}{\partial p}-q \frac{\partial}{\partial w} .
$$

We shall denote by $i T, i Q, i P$ the unbounded operators on $L^{2}(\mathcal{H})$, affiliated to $C^{*}(\mathcal{H})$, which correspond to these vector fields. Thus $P, Q, T$ are unbounded self-adjoint operators, which satisfy the commutation relation

$$
[P, Q]=-2 i T
$$




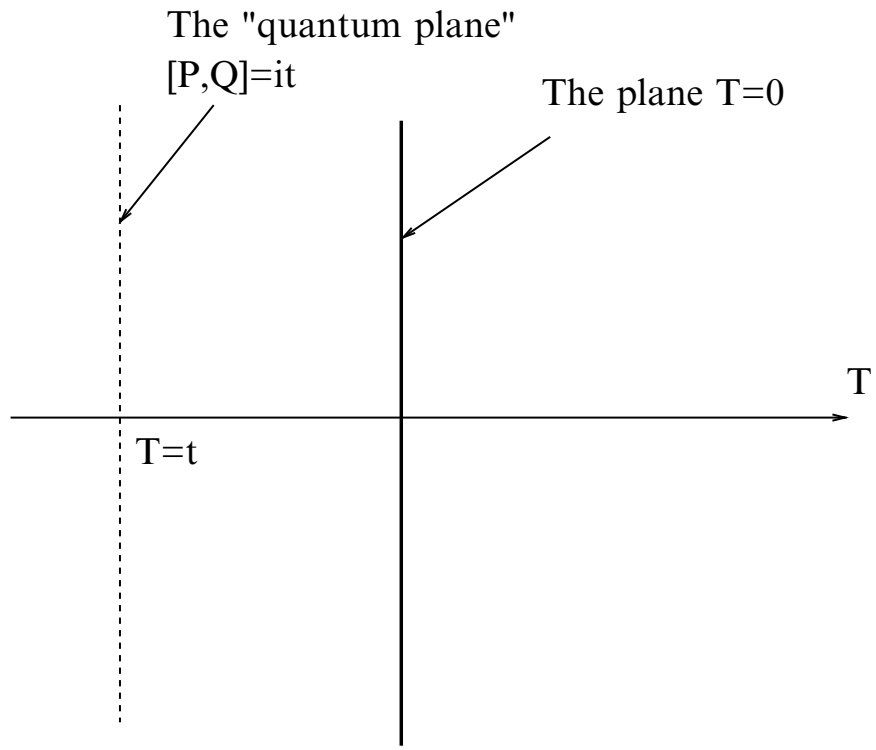

Fig. 5 The dual of the Heisenberg group.

As in the case of $S U(2)$ one can give a heuristic description of the noncommutative space dual to $\mathcal{H}$ using the generators of the Lie algebra of $\mathcal{H}$, which define three noncommuting unbounded self-adjoint operators $P, Q, T$. We think of these operators as coordinate functions on this dual space, satisfying the commutation relations

$$
[P, Q]=-2 i T \quad[P, T]=[Q, T]=0 .
$$

Since the coordinate $T$ belongs to the center, it allows to decompose the space into slices according to the values of this coordinate. When $T=0$, the coordinates $P$ and $Q$ commute, and the corresponding slice is a usual plane, with two real coordinates. This corresponds to the one dimensional representations of the group. When $T=\tau$ a non zero real number, the two coordinates $P, Q$ generate a von Neumann algebra isomorphic to $B(H)$, and corresponding to the irreducible representation sending $T$ to $\tau I$. Note that in this representation the operator $P^{2}+Q^{2}$ has a discrete spectrum $2|\tau|, 6|\tau|, 10|\tau|, \ldots$.

Let us consider, for $t \geq 0$, the functions on $\mathcal{H}$

$$
\varphi_{t}^{ \pm}(z, w)=\left\langle\rho_{ \pm t}(z, w) \Omega, \Omega\right\rangle=e^{ \pm i t w-\frac{1}{2} t z \bar{z}} .
$$

By construction, these functions are positive definite functions on $\mathcal{H}$, and form two multiplicative semigroups. To these functions correspond convolution semigroups of states, and semigroups of completely positive maps. 
The semigroup of noncommutative brownian motion on the dual of $\mathcal{H}$ is the associated semigroup of completely positive maps on $C^{*}(\mathcal{H})$. Recall that this semigroup is obtained by composing the coproduct $\Delta: C^{*}(\mathcal{H}) \rightarrow$ $M\left(C^{*}(\mathcal{H})\right) \otimes M\left(C^{*}(\mathcal{H})\right)$ (recall that $M(A)$ is the multiplier algebra of $A$ ) with the state associated with the function. Thus

$$
\Phi_{t}^{ \pm}=\left(\nu_{\varphi_{t}^{ \pm}} \otimes I\right) \circ \Delta
$$

or equivalently

$$
\Phi_{t}^{ \pm}\left(\lambda_{g}\right)=\varphi_{t}^{ \pm}(g) \lambda_{g} \quad \text { for } g \in \mathcal{H} .
$$

Let $\nu$ be a state on $C^{*}(\mathcal{H})$, and $\rho_{\nu}: C^{*}(\mathcal{H}) \rightarrow B\left(H_{\nu}\right)$ be the associated GNS representation. We consider the two families of homomorphisms

$$
\begin{gathered}
j_{t}^{ \pm}: C^{*}(\mathcal{H}) \rightarrow B\left(H_{\nu} \otimes \Gamma\left(L^{2}\left(\mathbb{R}_{+}\right)\right)\right) \\
j_{t}^{ \pm}(z, w)=\rho_{\nu}(z, w) \otimes W_{z 1_{[0, t]}} e^{ \pm i t w}
\end{gathered}
$$

We shall prove that these homomorphisms constitute dilations of some completely positive convolution semigroups on $C^{*}(\mathcal{H})$. For each time $t \geq 0$ we have a direct sum decomposition $L^{2}\left(\mathbb{R}_{+}\right)=L^{2}([0, t]) \oplus L^{2}([t,+\infty[)$ and a corresponding factorization

$$
\Gamma\left(L^{2}\left(\mathbb{R}_{+}\right)\right)=\Gamma\left(L^{2}([0, t])\right) \otimes \Gamma\left(L^{2}([t,+\infty[))\right.
$$

Accordingly for each $t>0$ there are subalgebras

$$
W_{t}=B\left(\Gamma\left(L^{2}([0, t])\right)\right) \otimes I \subset B\left(\Gamma\left(L^{2}\left(\mathbb{R}_{+}\right)\right)\right)
$$

and linear maps

$$
E_{t}:=I d \otimes\left\langle. \Omega_{[t}, \Omega_{[t}\right\rangle: B\left(\Gamma\left(L^{2}\left(\mathbb{R}_{+}\right)\right)\right) \rightarrow W_{t}
$$

where $\Omega_{[t}$ is the vacuum vector of the space $\Gamma\left(L^{2}([0, t])\right)$.

Lemma 11.1. For each $t \geq 0$ the map $E_{t}$ is a conditional expectation with respect to the state $\langle. \Omega, \Omega\rangle$.

Indeed if $a \in B\left(L^{2}\left(\mathbb{R}_{+}\right)\right)$has a decomposition $a=a_{t]} \otimes a_{[t}$ then one has

$$
E_{t}(a)=a_{t]} \otimes\left\langle a_{[t} \Omega_{[t}, \Omega_{[t}\right\rangle
$$

and for $b, c \in B\left(L^{2}([0, t])\right)$

$$
\begin{aligned}
\langle b a c \mathcal{E}(u), \mathcal{E}(v)\rangle & =\left\langle b E_{t}(a) c \mathcal{E}\left(u 1_{[0, t]}\right), \mathcal{E}\left(v 1_{1[0, t]}\right)\right\rangle\left\langle a_{[t} \mathcal{E}\left(u 1_{[t,+\infty}\right), E\left(v 1_{[t,+\infty[}\right)\right\rangle \\
& =\left\langle b E_{t}(a) c \mathcal{E}(u), \mathcal{E}(v)\right\rangle
\end{aligned}
$$

One checks easily that the homomorphism $j_{t}^{ \pm}$sends $C^{*}(\mathcal{H})$ to $W_{t}$, furthermore we have 
Proposition 11.2. The maps $\left(j_{t}^{ \pm}, E_{t}, W_{t}\right)$ form a dilation of the completely positive semigroup $\Phi_{t}^{ \pm}$, with initial distribution $\nu$.

Proof. This is a bookkeeping exercise using the definition of the Weyl operators, one has to check that, for $t, s \geq 0$ and $u, v \in L^{2}\left(\mathbb{R}_{+}\right)$, one has

$$
\left\langle j_{t+s}^{ \pm}(z, w) \mathcal{E}\left(u 1_{[0, t]}\right), \mathcal{E}\left(v 1_{[0, t]}\right)\right\rangle=e^{ \pm i s w-\frac{1}{2} s|z|^{2}}\left\langle j_{t}^{ \pm}(z, w) \mathcal{E}\left(u 1_{[0, t]}\right), \mathcal{E}\left(v 1_{[0, t]}\right)\right\rangle
$$

For every one parameter subgroup of $\mathcal{H}$, there is a completely positive semigroup given by restriction of $\left(\Phi_{t}^{ \pm}\right)_{t>0}$. For subgroups of the form $(x z, 0) ; u \in \mathbb{R}$, with $z \in \mathbb{C}^{*}$ isomorphic to $\mathbb{R}$, one sees that the semigroup is that of Brownian motion on the dual group. Thus we recover the Brownian motions $\left(P_{t}, Q_{t}\right)$ of the preceding section by looking at $j_{t}(P)$ and $j_{t}(Q)$.

The restriction to the center $(0, w) ; w \in \mathbb{R}$ gives a semigroup of uniform translation on the real line.

\subsection{The Quantum Bessel Process}

Bessel processes are radial parts of Brownian motions. Here we shall exploit the action of the unitary group $U(1)$ on the Heisenberg group in order to find an abelian algebra which is left invariant by the semigroup and study the Markov process associated with the restriction of $\Phi_{t}^{ \pm}$to this subalgebra. Let $e^{i \theta}$ be a complex number with modulus 1 , then there exists an automorphism $a_{\theta}$ of the Heisenberg group defined by

$$
a_{\theta}(z, w)=\left(e^{i \theta} z, w\right)
$$

and this automorphism extends to an automorphism of the $C^{*}$ algebra.

Proposition 11.3. The subalgebra of $C^{*}(\mathcal{H})$ composed of elements invariant under the above action of $U(1)$ is an abelian $C^{*}$ algebra.

The characters of this abelian $C^{*}$-algebra have been computed by A. Koranyi, they are given by the formula

$$
\chi(f)=\int_{\mathcal{H}} \omega(g) f(g) d g
$$

for $f \in L^{1}(\mathcal{H})$, invariant under the action of $U(1)$, where $\omega$ belongs to the set of functions

$$
\left\{\omega_{\tau, m} \mid \tau \in \mathbb{R}^{*}, m \in \mathbb{N}\right\} \cup\left\{\omega_{\mu} \mid \mu \in \mathbb{R}_{+}\right\}
$$




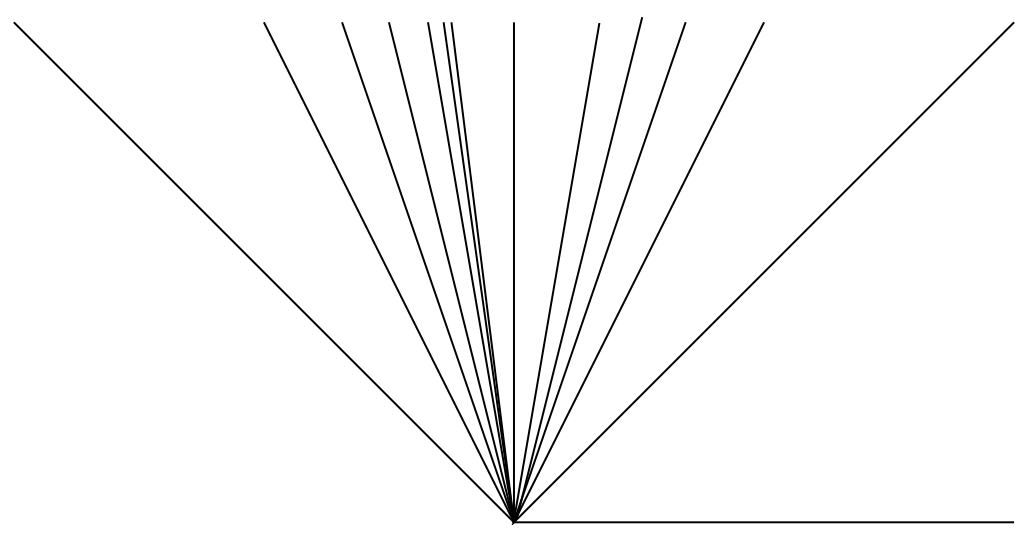

Fig. 6 The Heisenberg fan.

with

$$
\begin{aligned}
\omega_{\tau, m}(z, w) & =m e^{i \tau w-\frac{1}{2}|\tau||z|^{2}} L_{m}\left(|\tau||z|^{2}\right) \\
\omega_{\mu}(z, w) & =j_{0}\left(\mu|z|^{2}\right)
\end{aligned}
$$

Here $L_{m}$ are the Laguerre polynomials defined by the generating series

$$
\sum_{m=0}^{\infty} L_{m}(x) t^{m}=\frac{e^{-\frac{x t}{1-t}}}{1-t}
$$

and $j_{0}$ is the usual Bessel function.

The spectrum of the algebra $C_{R}^{*}(\mathcal{H})$ can be identified with a closed subset of $\mathbb{R}^{2}$, which consists in the union of the halflines $\{(x, k x) ; x>0\}$ for $k \in \mathbb{N}$, the halflines $(x, k x) ; x<0$ for $k \in \mathbb{N}^{*}$, and the halfline $(0, y) ; y \geq 0$.

It is the spectrum of the unbounded operator $\frac{1}{2}\left(P^{2}+Q^{2}-T\right)$, and this algebra is the algebra of functions of this operator.

The picture gives a "fan" consisting of a union of halflines originating from 0 as depicted below.

We shall call noncommutatif Bessel semigroup the restriction of $\Phi_{t}^{ \pm}$to the abelian subalgebra $C_{R}^{*}(\mathcal{H})$. In order to compute this semigroup we need, for each character $\omega$ to decompose the functions $\omega \varphi_{t}$ into an integral of characters. The result is given by the following.

Proposition 11.4. The noncommutative Bessel semigroup $\Phi_{t}^{+}$is given by the following kernel.

$$
\begin{aligned}
& \text { If } x=(\sigma,-k \sigma) \text { with } \sigma<0 \text { and } \tau=\sigma+t \text { then } \\
& \qquad q_{t}(x, d y)=\sum_{l=k}^{\infty} \frac{(l-1) !}{(k-1) !(l-k) !}\left(1-\frac{\tau}{\sigma}\right)^{l-k}\left(\frac{\tau}{\sigma}\right)^{k} \delta_{(\tau,-l \tau)}(d y)
\end{aligned}
$$




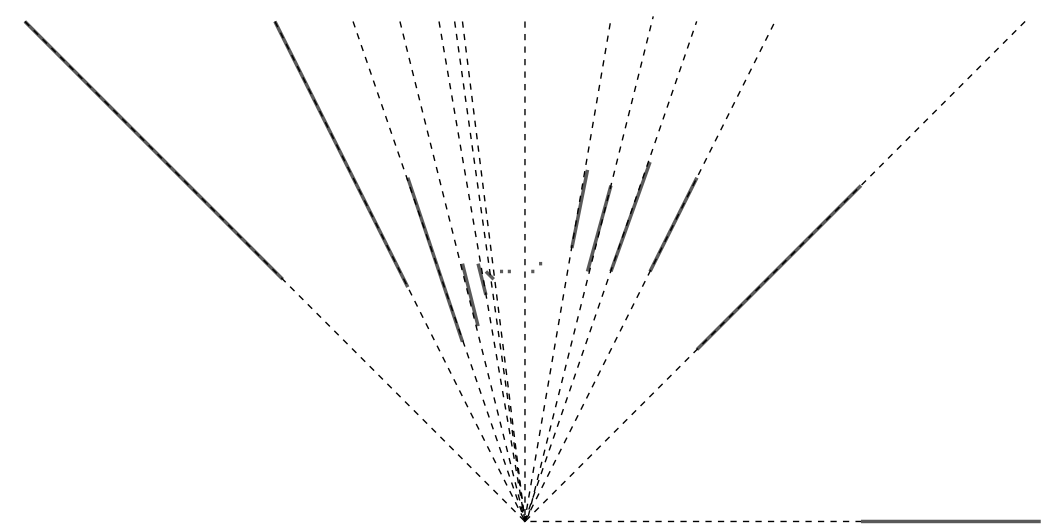

Fig. 7

$$
\text { If } x=(\sigma,-k \sigma) \text { with } \sigma<0 \text { and } 0=\sigma+t,(y=0, r) \text { then }
$$

$$
q_{t}(x, d y)=\frac{\left(\frac{r}{t}\right)^{k-1}}{(k-1) !} e^{-\frac{r}{t}} \delta_{0} \otimes d r
$$

If $x=(\sigma,-k \sigma)$ with $\sigma<0$ and $\tau=\sigma+>0$ then

$$
q_{t}(x, d y)=\sum_{l=0}^{\infty} \frac{(l+k-1) !}{(k-1) ! l !}\left(1-\frac{\tau}{t}\right)^{l+k}\left(\frac{\tau}{t}\right)^{k} \delta_{(\tau, l \tau)}(d y)
$$

If $x=(0, r)$ then

$$
q_{t}(x, d y)=\frac{\left(\frac{r}{t}\right)^{k-1}}{l !} e^{-\frac{t}{l t}} \delta_{t, l t}(d y)
$$

If $x=(\sigma, k \sigma)$ with $\sigma>0$ and $\tau=\sigma+t$

$$
q_{t}(x, d y)=\sum_{l=0}^{l=k} \frac{(l-1) !}{(k-1) !(l-k) !}\left(1-\frac{\sigma}{\tau}\right)^{k-l}\left(\frac{\sigma}{\tau}\right)^{l} \delta_{(\tau, l \tau)}(d y)
$$

The computations can be found in [B6].

A typical trajectory of the process is depicted in the above picture. It starts from a point $(\sigma,-\sigma)$ with $\sigma<0$. During the whole process the first coordinates follows a uniform translation to the right. The trajectory starts on the line $y=-x$, and with an intensity $\frac{d t}{-\sigma+t}$, then jumps to the line $(y=-2 x)$, which it follows before jumping to the next line $(y=-3 x)$ with an intensity $\frac{2 d t}{-\sigma+t}$, and so on, until it reaches after infinitely many jumps the line $x=0$, then the process on the right half plane does the jumps from the line $(y=k x)$ to $(y=(k-1) x)$, until it finally reaches the line $y=0$ where it stays forever. One can actually construct this process from a birth and death process known as the Yule process, and the embedding of this process into the Heisenberg fan yields a construction of the space-time boundary of this process, see $[\mathrm{B} 6]$. 


\section{Dilations for Noncompact Groups}

\subsection{The General Case}

We shall now extend the preceding construction to the case of arbitrary locally compact groups. Let $G$ be a locally compact group, with right Haar measure $m$, and consider its convolution algebra $L^{1}(G)$. We endow this algebra with the norm $\|f\|=\sup \|\rho(f)\|$ where the supremum is over all unitary representations $\rho$ of the group $G$, extended to the algebra $L^{1}(G)$. This yields the full $C^{*}$-algebra of $G$, denoted $C^{*}(G)$. When the group $G$ is amenable, for example if $G$ is compact or for the Heisenberg group which we have met before, this coincides with the completion of the action of the group on $L^{2}(G)$, which is called the reduced $C^{*}$-algebra of $G$. When the group is nonamenable, $C_{r}^{*}(G)$ is strictly smaller than $C^{*}(G)$. A continuous positive definite function on $G$, such that $\varphi(e)=1$ defines a state as well as a completely positive contraction on $C^{*}(G)$, whose restriction to $L^{1}(G)$ is given by $f \mapsto f \varphi$. Let now $\psi$ be a continuous, conditionally negative definite function on $G$, with $\psi(e)=0$. Recall that this means that for all $t \geq 0$ the function $e^{-t \psi}$ is a positive definite function on $G$, or equivalently, by Schönberg's theorem, that for all $z_{1}, \ldots, z_{n} \in \mathbb{C}$ with $\sum_{i} z_{i}=0$, and $g_{1}, \ldots, g_{n} \in G$ one has

$$
\sum_{i j} z_{i} \bar{z}_{j} \psi\left(g_{j}^{-1} g_{i}\right) \leq 0 .
$$

There is an associated semigroup of completely positive contractions on $C^{*}(G)$. We shall now, following Parthasarathy and Schmidt [PS], construct a dilation of this semigroup. Let $\nu$ be a state on $C^{*}(G)$ which will be the initial state. The GNS construction yields a unitary representation $\pi$ of $G$ on a Hilbert space $H_{\pi}$, and $\eta \in H_{\pi}$ such that $\nu(x)=\langle\pi(x) \eta, \eta\rangle$ for $x \in C^{*}(G)$. A variant of the GNS construction associates to the function $\psi$ a unitary representation of $G$ in a Hilbert space $H_{\psi}$, and a cocycle $v: G \rightarrow H_{\psi}$ for this representation. Thus $v$ is a continuous function which satisfies

$$
v(g h)=g v(h)+v(g)
$$

and

$$
\langle v(g), v(h)\rangle=-\psi\left(h^{-1} g\right)+\psi(g)+\psi\left(h^{-1}\right)-\psi(e)
$$

for all $g, h \in G$. Conversely, any function $\psi$ satisfying the above equation for some representation and cocycle $v$ is conditionally negative definite. Indeed one has

$$
\sum_{i j} z_{i} \bar{z}_{j} \psi\left(g_{j}^{-1} g_{i}\right)=-\left\|\sum_{j} z_{j} v\left(g_{j}\right)\right\|^{2} \leq 0
$$

if $\sum_{j} z_{j}=0$. 
Let $\Gamma=\Gamma\left(L^{2}\left(\mathbb{R}_{+}\right) \otimes H_{\psi}\right)$, and let $\mathcal{W}=B\left(H_{\pi} \otimes \Gamma\right) \sim B\left(H_{\pi}\right) \otimes B(\Gamma)$. Let $\omega$ be the pure state on $\mathcal{W}$ associated with the vector $\eta \otimes \Omega$. One can define the subalgebras $\mathcal{W}_{t}=B\left(H_{\pi} \otimes \Gamma_{t}\right) \otimes I d$ associated with the orthogonal decomposition $L^{2}\left(\mathbb{R}_{+}\right) \otimes H_{\psi}=\left(L^{2}([0, t]) \otimes H_{\psi}\right) \oplus\left(L^{2}\left(\left[t,+\infty[) \otimes H_{\psi}\right)\right.\right.$, and the conditional expectations $E_{t}: \mathcal{W} \rightarrow \mathcal{W}_{t}$ with respect to the state $\omega$. One defines a unitary representation of $G$ on exponential vectors by

$$
V^{t}(g)(\mathcal{E}(u))=e^{t \psi(g)+\left\langle 1_{[0, t]} \otimes v_{t}(g), u\right\rangle} \mathcal{E}\left(U^{t}(g)(u)+1_{[0, t]} \otimes v_{t}(g)\right)
$$

One thus gets a representation of $G$ on $H_{\pi} \otimes \Gamma$ by taking the tensor product of $V^{t}$ with the representation $\pi$, and this yields a family of morphisms $j_{t}$ : $C^{*}(G) \rightarrow \mathcal{W}$.

Proposition 12.1. The family $\left(j_{t}, \mathcal{W}, \mathcal{W}_{t}, E_{t}, \omega\right)$ forms a dilation of the completely positive semigroup, with initial distribution $\nu$.

The proof is a bookkeeping exercise. This construction has been extended by Schürmann to a class of bialgebras, see [Sc], allowing him in particular to give a nice construction of the Azéma martingales.

\subsection{Free Groups}

Let now $F_{n}$ be a free group on $n$ generators $g_{1}, \ldots, g_{n}$. Each element of $F_{n}$ can be written in a unique way as a reduced word $w=g_{i_{1}}^{\varepsilon_{1}} \ldots g_{i_{k}}^{\varepsilon_{k}}$, where one has $\varepsilon_{j}= \pm 1$ for all $j$ and $i_{1} \neq i_{2} \neq i_{3} \ldots i_{k-1} \neq i_{k}$. For such an element one defines its length $l(w)=k$. This is the smallest integer $k$ such that $w$ can be expressed as a product of $k$ elements in the set $\left\{g_{1}, g_{1}^{-1}, g_{2}, \ldots, g_{n}, g_{n}^{-1}\right\}$.

Proposition 12.2. (Haagerup $[H]$ ) The function $l$ is conditionally negative definite on $F_{n}$.

Proof. Consider the Cayley graph of $F_{n}$ built on the generators. Thus this graph has as vertices the elements of $F_{n}$ and its edges are the pairs $\{g, h\}$ such that $h^{-1} g$ is a generator or the inverse of a generator. This Cayley graph is a regular tree in which each vertex has $2 n$ neighbours. For any $g \in F_{n}$ one can consider the unique shortest path in the graph between $I d$ and $g$. Let $E_{n}$ be the set of edges of the Cayley graph, endowed with the counting measure, then one defines $v(g) \in L^{2}\left(E_{n}\right)$ to be the indicator function of this shortest path from $I d$ to $g$ in the Cayley graph. Thus $v(g)(e)=1$ if and only if the edge $e$ is on the shortest path from $I d$ to $g$. One can easily check, using the properties of trees that for any $h, h \in F_{n}$ one has

$$
l(g)+l(h)-l\left(h^{-1} g\right)=2\langle v(g), v(h)\rangle_{L^{2}\left(F_{n}\right)}
$$


indeed this scalar product counts the number of common edges in the shortest paths from $I d$ to $g$ and $h$. This implies, by (12.1) that $l$ is conditionally negative definite.

It follows from the previous proposition that there exists, on the full $C^{*}$ algebra of $F_{n}$, a semigroup of unit preserving completely positive maps, given by the formula

$$
\Phi_{t}\left(\lambda_{g}\right)=e^{-t l(g)} \lambda_{g}
$$

The theory of the previous section allows us to construct a dilation of this semigroup. As before we shall be interested in the restriction of this completely positive semigroup to commutative subalgebras. The first one will be the subalgebra of the subgroup generated by one of the generators. Let $g_{i}$ be this generator, then this subgroup is isomorphic to $\mathbb{Z}$ by $k \mapsto g_{i}^{k}$, therefore its dual is isomorphic to the group of complex numbers of modulus 1 . The restriction gives us a Markov semigroup on the group of complex numbers of modulus 1. This semigroup is easy to characterize, it sends the function $e^{i k \theta}$ on the unit circle to the function $e^{-|k|+i k \theta}$. In other words this is the integral operator on the unit circle given by the Poisson kernel

$$
P_{t}\left(\theta, \theta^{\prime}\right)=\frac{1-e^{-2 t}}{1-2 e^{-t} \cos \left(\theta-\theta^{\prime}\right)+e^{-2 t}} .
$$

This is a convolution semigroup, as expected.

The other commutative algebra of interest is the algebra $\mathcal{R}\left(F_{n}\right)$ consisting of radial elements. It is generated by the elements $\chi_{l}=\sum_{l(g)=l} \lambda_{g}$ for $l=0,1, \ldots$, and it is immediate that the completely positive semigroup associated with the length function leaves this algebra invariant. Actually one has $\Phi_{t}\left(\chi_{l}\right)=e^{-t l} \chi_{l}$. These elements satisfy the relations

$$
\begin{aligned}
\chi_{0} & =I \\
\chi_{1}^{2} & =\chi_{2}+2 n \chi_{0} \\
\chi_{l} \chi_{l} & =\chi_{l+1}+(2 n-1) \chi_{l-1} \quad l \geq 2
\end{aligned}
$$

From this we conclude that $\mathcal{R}\left(F_{n}\right)$ is the commutative von Neumann algebra generated by the self-adjoint element $\chi_{1}$, and its spectrum is the spectrum of $\chi_{1}$. In order to compute the norm of $\chi_{l}$ we need just to consider the trivial representation of $F_{n}$ in which all $g_{i}$ are mapped to the identity, and we get $\left\|\chi_{l}\right\|=2 n(2 n-1)^{l-1}$ for $l \geq 1$, the number of elements of length $l$ in $F_{n}$. Any character $\varphi: \mathcal{R}\left(F_{n}\right) \rightarrow \mathbb{C}$ is determined by its values on $\chi_{1}$. For such a character $\varphi$, with $\varphi\left(\chi_{1}\right)=x$, one has

$\varphi\left(\chi_{0}\right)=1 ; \varphi\left(\chi_{2}\right)=x^{2}-2 n ; \quad \varphi\left(\chi_{l+1}\right)=x \varphi\left(\chi_{l}\right)-(2 n-1) \varphi\left(\chi_{l-1}\right)$ for $l \geq 2$

from which one infers that 


$$
\varphi\left(\chi_{l}\right)=\frac{\lambda_{1}^{l+1}-\lambda_{2}^{l+1}}{\lambda_{1}-\lambda_{2}}-\frac{\lambda_{1}^{l-1}-\lambda_{2}^{l-1}}{\lambda_{1}-\lambda_{2}} \quad \text { for } l \geq 1
$$

where $\lambda_{1}, \lambda_{2}$ are the two roots of the equation $\lambda^{2}-x \lambda+2 n-1=0$.

We verify that the character $\varphi_{x}$ defined by the formula above is real and bounded if and only if $x \in[-2 n, 2 n]$. The spectrum of the algebra $\mathcal{R}\left(F_{n}\right)$ thus coincides with the interval $[-2 n, 2 n]$, and the element $\chi_{l}$ corresponds to a polynomial function $P_{l}\left(\chi_{1}\right)=\chi_{l}$, where the polynomials are determined by the recursion

$$
P_{0}=1, \quad P_{1}(x)=x, \quad x P_{l}(x)=P_{l+1}(x)+(2 n-1) P_{l-1} .
$$

This three term recursion relation is characteristic of a sequence of orthogonal polynomials. The orthogonalizing measure is the distribution of $\chi_{1}$ in the noncommutative probability space $\left(\mathcal{A}\left(F_{n}\right), \delta_{e}\right)$ where $\delta_{e}$ is the pure state, in the left regular representation of $F_{n}$, corresponding to the identity. Thus $\delta_{e}\left(\lambda_{g}\right)=1$ if $g=e$ and $\delta_{e}\left(\lambda_{g}\right)=0$ if not. This measure is known as the Kesten measure (see $[\mathrm{Ke}]$ ) and has the density

$$
d m_{n}(x)=\frac{2 n}{2 n-1} 4 \pi \frac{\sqrt{4(2 n-1)-x^{2}}}{4 n^{2}-x^{2}}
$$

on the interval $[-2 \sqrt{2 n-1}, 2 \sqrt{2 n-1}]$. The discrepancy between the interval $[-2 n, 2 n]$ which is the spectrum of $\chi_{1}$ and the support of the measure $m_{n}$ comes from the fact that $F_{n}$ is a nonamenable group and therefore some of its unitary representations are not weakly contained in the regular representation.

The semigroup of the restriction of $\left(\Phi_{t}\right)_{t \geq 0}$ to the subalgebra $\mathcal{R}\left(F_{n}\right)$ sends the polynomial function $P_{l}(x)$ on the interval $[-2 n, 2 n]$ to the function $e^{-t l} P_{l}(x)$. We can compute the transition probabilities $p_{t}(x, d y)$ by finding the integral representation

$$
e^{-t l} P_{l}(x)=\int_{[-2 n, 2 n]} p_{t}(x, d y) P_{l}(y)
$$

for each $x \in[-2 n, 2 n]$.

If $x$ belongs to the support of the Kesten measure, then since the polynomials $P_{l} ; l \geq 0$ form an orthogonal basis of the $L^{2}$ space of the Kesten measure, and $\left\|P_{l}\right\|_{2}^{2}=2 n(2 n-1)^{l}$ one obtains $p_{t}$ through the orthogonal expansion

$$
p_{t}(x, d y)=e^{-t} d m_{n}(y)+\sum_{l=1}^{\infty} e^{-t l} \frac{1}{2 n(2 n-1)^{l-1}} P_{l}(x) P_{l}(y) d m_{n}(y)
$$

When $x$ is outside this support, then by (12.2) the sequence $P_{l}(x)$ is unbounded and has exponential growth of rate $\xi=\frac{x+\sqrt{x^{2}-4(2 n-1)}}{2}$ and the 
character value $e^{-t l} P_{l}(x)$ has the asymptotic behaviour $e^{-t l} \xi^{l}$ as $l \rightarrow \infty$, and if $e^{-t} \xi>2 \sqrt{2 n-1}$, then let $x(t)=e^{-t} \xi+(2 n-1) e^{t} / \xi$. The function $e^{-t l} P_{l}(x)$ can be written as is a linear combination of $P_{l}(x(t))$, which picks up the dominant term as $l \rightarrow \infty$ and the $P_{l}(y)$ for $y$ in the interval $[-2 \sqrt{2 n-1}, 2 \sqrt{2 n-1}]$. More precisely the quantity

$$
Q_{l}^{t}(x)=e^{-t l} P_{l}(x)-\frac{e^{-t} \xi-(2 n-1) e^{t} / \xi}{e^{-t} \xi-e^{t} / \xi} \frac{\xi-(2 n-1) / \xi}{\xi-1 / \xi} P_{l}(x(t))
$$

decreases exponentially as $l \rightarrow \infty$, and thus one has

$$
p_{t}(x, d y)=c_{t} \delta_{x(t)}+\sum_{l=1}^{\infty} e^{-t l} \frac{1}{2 n(2 n-1)^{l-1}}\left(P_{l}(x)-c_{t} P_{l}(x(t))\right) P_{l}(y) d m_{n}(y)
$$

with $c_{t}=\frac{e^{-t} \xi-(2 n-1) e^{t} / \xi}{e^{-t} \xi-e^{t} / \xi} \frac{\xi-(2 n-1) / \xi}{\xi-1 / \xi}$. If $\xi(t)<2 \sqrt{2 n-1}$ then there is a similar decomposition, but the term $c_{t}$ is 0 .

Thus the process starting form a point $x \in[-2 n, 2 n] \backslash[-2 \sqrt{2 n-1}$, $2 \sqrt{2 n-1}]$ performs a translation towards the central interval, and at some point jumps into it, and after that performs a certain pure jump process inside the interval $[-2 \sqrt{2 n-1}, 2 \sqrt{2 n-1}]$ where it remains forever.

\section{Pitman's Theorem and the Quantum Group $S U_{q}(2)$}

\subsection{Pitman's Theorem}

Let $\left(B_{t}\right)_{t \geq 0}$ be a real Brownian motion, with $B_{0}=0$, and let

$$
S_{t}=\sup _{0 \leq s \leq t} B_{s}
$$

then Pitman's theorem states that the stochastic process

$$
R_{t}=2 S_{t}-B_{t} ; \quad t \geq 0
$$

is a three dimensional Bessel process, i.e. is distributed as the norm of a three dimensional Brownian motion. There is a discrete version of Pitman's theorem, actually it is this discrete version that Pitman proved in his original paper $[\mathrm{Pi}]$. We start from a symmetric Bernoulli random walk $X_{n}=x_{1}+\ldots+$ $x_{n}$ where the $x_{i}$ are i.i.d. with $P\left(x_{i}\right)= \pm 1=1 / 2$, and build the processes $S_{n}=\max _{1 \leq k \leq n} X_{k}$, and $T_{n}=2 S_{n}-X_{n}$. Pitman proved in [Pi] that $\left(T_{n}\right)_{n \geq 0}$ is a Markov chain on the nonegative integers, with probability transitions 


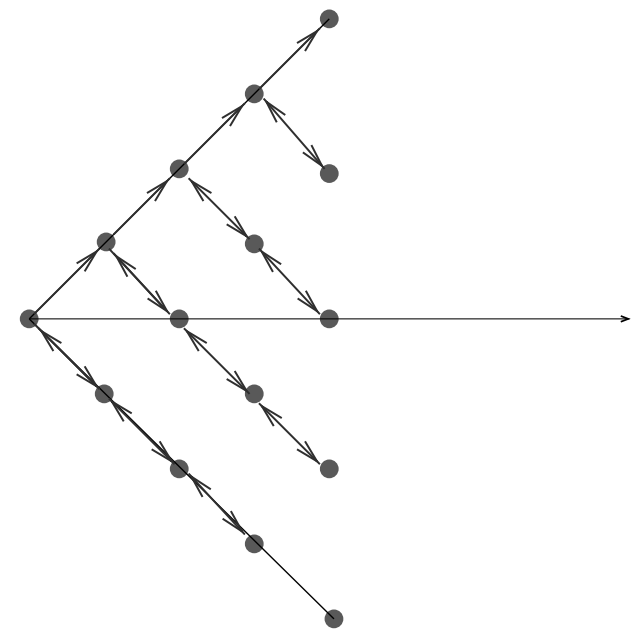

Fig. 8

$$
p(k, k+1)=\frac{k+2}{2(k+1)} \quad p(k, k-1)=\frac{k}{2(k+1)}
$$

and the theorem about Brownian motion can be obtained by taking the usual approximation of Brownian motion by random walks. We see that this Markov chain is, up to a shift of 1 in the variable, exactly the one that we obtained in Theorem 3.2 when considering the spin process. This is not a coincidence as we shall see, actually we will understand this connection by introducing quantum groups in the picture. Before that, let us give the proof of Pitman's theorem We consider the stochastic process $\left(\left(S_{n}, X_{n}\right) ; n \geq 1\right)$, with values in $\{(s, k) \in \mathbb{N} \times \mathbb{Z} \mid s \geq k\}$. It is easy to see that this stochastic process is a Markov chain, with transition probabilities

$$
\begin{array}{cc}
p((s, k),(s, k+1))=\frac{1}{2}, \quad p((s, k),(s, k-1))=\frac{1}{2} & \text { for } s>k \\
p((s, s),(s+1, s+1))=\frac{1}{2}, & p((s, s),(s, s-1))=\frac{1}{2}
\end{array}
$$

from which we can deduce the probability transitions of the Markov chain $\left(\left(T_{n}, X_{n}\right) ; n \geq 1\right)$, with values in $\left\{(t, k) \in \mathbb{N}^{*} \times \mathbb{Z} \mid k \in(-t,-t+2, \ldots, t-2, t)\right\}$,

$$
\begin{aligned}
& p((t, k),(t-1, k+1))=\frac{1}{2}, \quad p((t, k),(t+1, k-1))=\frac{1}{2} \quad \text { if } t>k \\
& p((t, t),(t+1, t+1))=\frac{1}{2}, \quad p((t, t),(t+1, t-1))=\frac{1}{2}
\end{aligned}
$$

The transition probabilities are depicted in this picture.

One checks then, by induction on $n$, that the conditional distribution of $X_{n}$, knowing $T_{1}, \ldots, T_{n}$, is the uniform distribution on the set $\left\{-T_{n},-T_{n}+\right.$ $\left.2, \ldots, T_{n}-2, T_{n}\right\}$. Then it follows that $\left(T_{n} ; n \geq 0\right)$ is a Markov chain with the right transition probabilities. 


\subsection{A Markov Chain Associated with the Quantum Bernoulli Random Walk}

In section 5.2 we have seen that the quantum Bernoulli random walk gives rise in a natural way to two Markov chains, one being the classical Bernoulli random walk, and the other being the spin process. These two processes were obtained in the preceding section as coordinates of a certain twodimensional Markov chain given by the transition probabilities (13.1). We can also consider a two-dimensional Markov chain having these two processes as marginals, by considering the Markov chain of the end of section 5.2. Recall that this Markov chain was obtained by restricting the generator of the quantum Bernoulli random walk to the commutative subalgebra $\mathcal{P}(S U(2)) \subset \mathcal{A}(S U(2))$ generated by the center $\mathcal{Z}(S U(2))$ and by a one parameter subgroup. The spectrum of this algebra can be identified with the set

$$
\hat{P}=\{(r, k) \in \mathbb{N} \times \mathbb{Z} \mid k \in\{-r,-r+2, \ldots, r-2, r\}\}
$$

Indeed this algebra is generated by the pair of commuting self-adjoint operators $X, D$ in the sense that it consists in bounded functions of the pair $(X, D)$. The joint spectrum of these operators can be computed from the explicit description of the irreducible representations of $S U(2)$, and coincides with $\hat{P}$. The probability transitions can be obtained by using the ClebschGordan formula, or equivalently by the computation in the proof of Lemma 3.3. One finds

$$
\begin{aligned}
& p((r, k),(r+1, k+1))=\frac{r+k+2}{2(r+1)} \\
& p((r, k),(r+1, k-1))=\frac{r-k+2}{2(r+1)} \\
& p((r, k),(r-1, k+1))=\frac{r-k}{2(r+1)} \\
& p((r, k),(r-1, k-1))=\frac{r+k}{2(r+1)} .
\end{aligned}
$$

These transition probabilities are on this picture

Thus, although this Markov chain has the same one-dimensional marginal as the one of the preceding section, they do not coincide. We will see that in order to recover the transitions (13.1) we will have to introduce quantum groups.

\subsection{The Quantum Group $S U_{q}(2)$}

The Hopf algebra $\mathcal{A}(S U(2))$ can be deformed by introducing a real parameter $q$. The algebraic construction proceeds with the introduction of three 


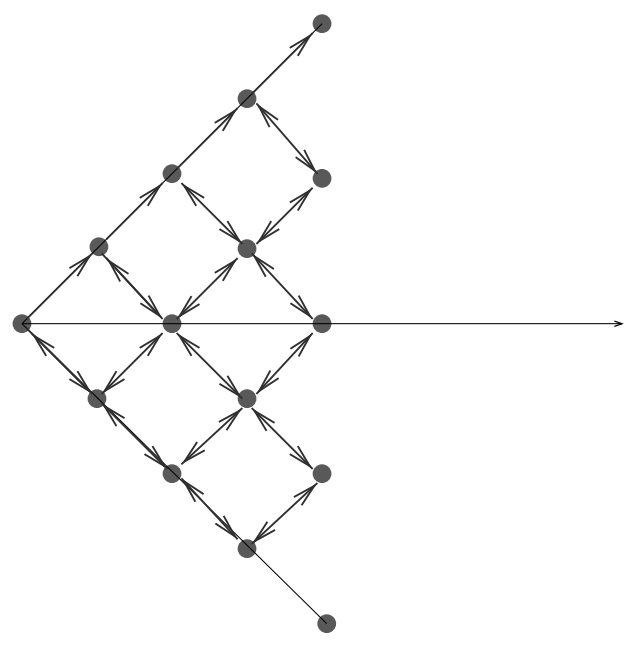

Fig. 9

generators $t, e, f$ which are required to satisfy the relations

$$
t e t^{-1}=q^{2} e, \quad t f t^{-1}=q^{-2} f, \quad \text { ef }-f e=\frac{t-t^{-1}}{q-q^{-1}}
$$

and a coproduct which is given by

$$
\Delta(t)=t \otimes t, \quad \Delta(e)=e \otimes t^{-1}+1 \otimes e, \quad \Delta(f)=f \otimes 1+t \otimes f
$$

Formally putting $t=q^{h}$ and letting $q$ converge to 1 one finds in the limit the defining relations for the envelopping algebra of the Lie algebra of $S U(2)$, as well as the coproduct.

One can prove that the irreducible finite dimensional representations of this algebra are deformations of those of $S U(2)$, indeed for each integer $r \geq 0$ there exists two representation in $V_{r+1}^{+}$and $V_{r+1}^{-}$, with bases $v_{k}^{r \pm} ; k \in\{-r,-r+2, \ldots, r-2, r\}$, given by

$$
\begin{aligned}
t v_{j}^{r \pm} & = \pm q^{j} v_{j}^{r \pm} \\
e v_{j}^{r \pm} & = \pm \sqrt{\left[\frac{r-j}{2}\right]_{q}\left[\frac{r+j+2}{2}\right]_{q}} v_{j+2}^{r \pm} \\
f v_{j}^{r \pm} & =\sqrt{\left[\frac{r-j+2}{2}\right]_{q}\left[\frac{r+j}{2}\right]_{q}} v_{j-2}^{r \pm} .
\end{aligned}
$$

with $[n]_{q}=\frac{q^{n}-q^{-n}}{q-q^{-1}}$. Using the coproduct one can define the tensor product of two representations, and this tensor product obeys the same rules as the 
one for representations of $S U(2)$ i.e. one has

$$
V_{r_{1}+1}^{\epsilon_{1}} \otimes V_{r_{2}+1}^{\epsilon_{2}}=\bigoplus_{r=\left|r_{2}-r_{1}\right|,\left|r_{2}-r_{1}\right|+2, \ldots, r_{1}+r_{2}} V_{r+1}^{\epsilon_{1} \epsilon_{2}} .
$$

We will now restrict our attention to representations of the kind $V_{l}^{+}$, and consider the von Neumann-Hopf algebra $\mathcal{A}^{+}\left(S U_{q}(2)\right)=\oplus_{r \geq 0} \operatorname{End}\left[V_{r+1}^{+}\right]$, which is isomorphic, as an algebra, to $\mathcal{A}(S U(2))$, but whose coproduct is deformed. The subalgebra $\mathcal{P}\left(S U_{q}(2)\right)$ generated by $t$ and by the center remains unchanged in the deformation. We consider the tracial state $\frac{1}{2} \operatorname{Tr}$ on the twodimensional component, and consider the associated random walk. As in the case of $S U(2)$, the restriction of the associated Markov transtion operator to the commutative algebra $\mathcal{P}\left(S U_{q}(2)\right)$ defines a Markov chain on the spectrum of this algebra, whose transition probabilities can be computed, using the deformed Clebsch Gordan formulas, as in Klimyk et Vilenkin [KV], formulas (6) et $(9), \S 14.4 .3$, to give

$$
\begin{aligned}
& p((r, k),(r+1, k+1))=q^{(r-k) / 2} \frac{\left[\frac{r+k+2}{2}\right]_{q}}{[r+1]_{q}}=\frac{q^{r+1}-q^{-k-1}}{2\left(q^{r+1}-q^{-r-1}\right)} \\
& p((r, k),(r+1, k-1))=q^{-(r+k) / 2} \frac{\left[\frac{r-k+2}{2}\right]_{q}}{2[r+1]_{q}}=\frac{q^{-k+1}-q^{-r-1}}{2\left(q^{r+1}-q^{-r-1}\right)} \\
& p((r, k),(r-1, k+1))=q^{-(r+k+2) / 2} \frac{\left[\frac{r-k}{2}\right]_{q}}{2[r+1]_{q}}=\frac{q^{-k-1}-q^{-r-1}}{2\left(q^{r+1}-q^{-r-1}\right)} \\
& p((r, k),(r-1, k-1))=q^{(r-k+2) / 2} \frac{\left[\frac{r+k}{2}\right]_{q}}{2[r+1]_{q}}=\frac{q^{r+1}-q^{-k+1}}{2\left(q^{r+1}-q^{-r-1}\right)}
\end{aligned}
$$

Letting $q$ tend to 0 , one checks that (13.2) converges to (13.1), and thus we get Pitman's theorem as an outcome of the $q \rightarrow 0$ limit of the quantum Bernoulli random walk, see [B7] for details.

This observation is at the basis of a vast generalization of Pitman's theorem, see e.g. [BBO].

\section{References}

[B1] P. Biane, Some properties of quantum Bernoulli random walks. Quantum probability \& related topics, 193-203, QP-PQ, VI, World Sci. Publ., River Edge, NJ, 1991.

[B2] P. Biane, Quantum random walk on the dual of SU(n). Probab. Theory Related Fields 89 (1991), no. 1, 117-129.

[B3] P. Biane, Minuscule weights and random walks on lattices. Quantum probability \& related topics, 51-65, QP-PQ, VII, World Sci. Publ., River Edge, NJ, 1992.

[B4] P. Biane, Équation de Choquet-Deny sur le dual d'un groupe compact. Probab. Theory Related Fields 94 (1992), no. 1, 39-51. 
[B5] P. Biane, Théorème de Ney-Spitzer sur le dual de SU(2). Trans. Amer. Math. Soc. 345 (1994), no. 1, 179-194.

[B6] P. Biane, Quelques propriétés du mouvement brownien non-commutatif. Hommage à P. A. Meyer et J. Neveu. Astérisque No. 236 (1996), 73-101.

[B7] P. Biane, Le théorème de Pitman, le groupe quantique $\mathrm{SU}_{q}(2)$, et une question de P. A. Meyer. In memoriam Paul-André Meyer: Séminaire de Probabilités XXXIX, 61-75, Lecture Notes in Math., 1874, Springer, Berlin, 2006.

[BBO] P. Biane, P. Bougerol, N. O'Connell, Littelmann paths and Brownian paths. Duke Math. J. 130 (2005), no. 1, 127-167.

[BtD] T. Bröcker, T. tom Dieck, Representations of compact Lie groups. Graduate Texts in Mathematics, 98. Springer-Verlag, New York, 1985.

[Col] B. Collins, Martin boundary theory of some quantum random walks. Ann. Inst. H. Poincaré Probab. Statist. 40 (2004), no. 3, 367-384.

[C] A. Connes, Noncommutative geometry. Academic Press, 1995.

[CD] G. Choquet, J. Deny, Sur l'équation de convolution $\mu=\mu * \sigma$. C. R. Acad. Sci. Paris $2501960799-801$.

[D1] J. Dixmier Les $C^{*}$-algèbres et leurs représentations. Gauthier-Villars. Paris, 1969.

[D2] J. Dixmier Les algèbres d'opérateurs dans l'espace hilbertien (algèbres de von Neumann). Gauthier-Villars, Paris, 1969.

[GW] R. Goodman, N.R. Wallach, Representations and invariants of the classical groups. Encyclopedia of Mathematics and its Applications, 68. Cambridge University Press, Cambridge, 1998.

[H] U. Haagerup, An example of a nonnuclear $C^{*}$-algebra, which has the metric approximation property. Invent. Math. 50 (1978/79), no. 3, 279-293.

[I] M. Izumi, Non-commutative Poisson boundaries and compact quantum group actions. Adv. Math. 169 (2002), no. 1, 1-57.

[INT] M. Izumi, S. Neshveyev, L. Tuset, Poisson boundary of the dual of $\mathrm{SU}_{q}(n)$. Comm. Math. Phys. 262 (2006), no. 2, 505-531.

[K] M. Kashiwara, Bases cristallines des groupes quantiques. Rédigé par C. Cochet. Cours Spcialisés, 9. Société Mathmatique de France, Paris (2002).

[Ke] H. Kesten, Symmetric random walks on groups. Trans. Amer. Math. Soc. 921959 336-354.

[KKS] J.G. Kemeny, J.L. Snell, A.W. Knapp, Denumerable Markov chains. Second edition. With a chapter on Markov random fields, by David Griffeath. Graduate Texts in Mathematics, No. 40. Springer-Verlag, New York-Heidelberg-Berlin, 1976.

[KV] A.U. Klimyk, N.Ja. Vilenkin, Representation of Lie groups and special functions. Vol. 2. Class I representations, special functions, and integral transforms. Mathematics and its Applications (Soviet Series), 74. Kluwer Academic Publishers Group, Dordrecht (1993).

[NS] P. Ney, F. Spitzer, The Martin boundary for random walk. Trans. Amer. Math. Soc. 1211966 116-132.

[Pa] K.R. Parthasarathy, An introduction to quantum stochastic calculus. Monographs in Mathematics, 85. Birkhuser Verlag, Basel, 1992.

[Pi] J.W. Pitman, One-dimensional Brownian motion and the three-dimensional Bessel process. Advances in Appl. Probability 7 (1975), no. 3, 511-526.

[PS] K.R. Parthasarathy, K. Schmidt, Positive definite kernels, continuous tensor products, and central limit theorems of probability theory. Lecture Notes in Mathematics, Vol. 272. Springer-Verlag, Berlin-New York, 1972.

[R] D. Revuz, Markov chains. Second edition. North-Holland Mathematical Library, 11. North-Holland Publishing Co., Amsterdam, 1984.

[Rie] M.A. Rieffel, Gromov-Hausdorff distance for quantum metric spaces. Matrix algebras converge to the sphere for quantum Gromov-Hausdorff distance. Mem. Amer. Math. Soc. 168 (2004), no. 796. American Mathematical Society, Providence, RI, 2004. 
[S] J.L. Sauvageot, Markov quantum semigroups admit covariant Markov $C^{*}$ dilations. Comm. Math. Phys. 106 (1986), no. 1, 91-103.

[Sc] M. Schürmann, White noise on bialgebras. Lecture Notes in Mathematics, 1544. Springer-Verlag, Berlin, 1993.

[T] M. Takesaki, Theory of operator algebras. I, II, III. Encyclopaedia of Mathematical Sciences, 124, 125, 127 Operator Algebras and Non-commutative Geometry, 5, 6, 8. Springer-Verlag, Berlin, 2002/2003.

[Z] D.P. Želobenko, Compact Lie groups and their representations. Translated from the Russian by Israel Program for Scientific Translations. Translations of Mathematical Monographs, Vol. 40. American Mathematical Society, Providence, R.I., 1973. 